\title{
ON CERTAIN ISOGENIES BETWEEN K3 SURFACES
}

\author{
CHIARA CAMERE AND ALICE GARBAGNATI
}

\begin{abstract}
The aim of this paper is to construct "special" isogenies between K3 surfaces, which are not Galois covers between K3 surfaces, but are obtained by composing cyclic Galois covers, induced by quotients by symplectic automorphisms. We determine the families of K3 surfaces for which this construction is possible. To this purpose we will prove that there are infinitely many big families of K3 surfaces which both admit a finite symplectic automorphism and are (desingularizations of) quotients of other K3 surfaces by a symplectic automorphism.

In the case of involutions, for any $n \in \mathbb{N}_{>0}$ we determine the transcendental lattices of the K3 surfaces which are $2^{n}: 1$ isogenous (by a non Galois cover) to other K3 surfaces. We also study the Galois closure of the $2^{2}: 1$ isogenies and we describe the explicit geometry on an example.
\end{abstract}

\section{INTRODUCTION}

K3 surfaces are symplectic regular surfaces and among their finite order automorphisms the ones which preserve the symplectic structure (the symplectic automorphisms) play a special role. Indeed, the quotient of a K3 surface by a finite symplectic automorphism produces a singular surface whose desingularization is again a K3 surface. This construction establishes a particular relation between different sets of K3 surfaces: the ones which admit a finite symplectic automorphism and the ones obtained as desingularization of the quotient of a K3 surfaces by a symplectic automorphism. In the following the latter K3 surfaces are said to be (cyclically) covered by a K3 surface and the former are said to be the cover of a K3 surface. We denote by $\mathcal{L}_{n}$ the set of the K3 surfaces which admit an order $n$ symplectic automorphism and by $\mathcal{M}_{n}$ the set of the K3 surfaces which are $n: 1$ cyclically covered by a K3 surface. From now on we assume the surfaces to be projective.

Thanks to several works, starting from the end of the 70's until now (see, e.g. [N2, Mo, vGS, GSar1, GSar2 GSar3, G2]), the sets $\mathcal{L}_{n}$ and $\mathcal{M}_{n}$ are described as the union of countably many families of $R$ polarized K3 surfaces, for certain known lattices $R$. The dimension of these families is at most 11, and, recalling that the families of generic projective K3 surfaces have dimension 19, one immediately observes that the K3 surfaces which either admit a finite symplectic automorphism or which are cyclically covered by a K3 surface are quite special. So, it is natural to expect that the intersection $\mathcal{L}_{n} \cap \mathcal{M}_{n}$ is extremely small, i.e. that a K3 surface which is both covered and cover of another K3 surface is really rare. On the other hand, there is at least one known example of a family of K3 surfaces

2010 Mathematics Subject Classification. Primary 14J28, 14J50; Secondary 14J10.

Key words and phrases. K3 surfaces, Quotients, Symplectic automorphisms on K3 surfaces, Galois covers between K3 surfaces, Isogenies between K3 surfaces. 
contained in $\mathcal{L}_{n} \cap \mathcal{M}_{n}$, given by the family of the K3 surfaces which admit an elliptic fibration with an $n$-torsion section (see Section 3). This family has codimension one in the families which are components of $\mathcal{L}_{n}$ and of $\mathcal{M}_{n}$. Hence, surprisingly, the intersection $\mathcal{L}_{n} \cap \mathcal{M}_{n}$ is not so small.

The aim of this paper is to investigate more precisely the intersection between the two sets $\mathcal{L}_{n}$ and $\mathcal{M}_{n}$ and to relate it with the study of isogenies between K3 surfaces. In this paper, the term "isogeny between K3 surface" means a generically finite rational map between K3 surfaces, as in [I] and [BSV].

The quotient by a finite symplectic automorphism on a K3 surface $X$ induces an isogeny between $X$, which admits the symplectic automorphism, and the K3 surface $Y$ cyclically covered by $X$. The isogeny is birationally the quotient map and has of course the same order as the automorphism. There are other isogenies between K3 surfaces, which are not quotient maps, see e.g. [I] and [BSV]. Here we discuss one of these other isogenies: given a K3 surface $Z \in \mathcal{L}_{n} \cap \mathcal{M}_{n}$, it induces an $n^{2}: 1$ isogeny between other two K3 surfaces. Indeed, since $Z \in \mathcal{M}_{n}$, it is $n: 1$ covered by a K3 surface $X$; since $Z \in \mathcal{L}_{n}$, it is an $n: 1$ cover of a K3 surface $Y$. By composing these two $n: 1$ maps one obtains an $n^{2}: 1$ isogeny between $X$ and $Y$. We will prove that generically this isogeny is not induced by a quotient map.

In Section 2 we recall some preliminary results on the set $\mathcal{L}_{n}$ of K3 surfaces admitting a symplectic automorphism of order $n$ and on the set $\mathcal{M}_{n}$ of the K3 surfaces $n: 1$ cyclically covered by a K3 surface. In Section 3 we obtain our main results on the intersection $\mathcal{L}_{n} \cap \mathcal{M}_{n}$. In particular in Theorem 3.9 we prove:

Theorem There are components $\mathcal{Z}$ of $\mathcal{L}_{n} \cap \mathcal{M}_{n}$ such that $\operatorname{dim}\left(\mathcal{L}_{n}\right)=\operatorname{dim}\left(\mathcal{M}_{n}\right)=$ $\operatorname{dim} \mathcal{Z}$, i.e. the dimension of $\mathcal{Z}$ is the maximal possible and thus $\mathcal{Z}$ is an irreducible component of both of $\mathcal{L}_{n}$ and $\mathcal{M}_{n}$.

As a consequence we construct $n^{2}: 1$ isogenies and we prove that generically they are not quotient maps. The Section 4 contains the main results for the case $n=2$. In addition to the results which hold for every admissible $n$, we also obtain the following theorem (see Theorem 4.6 and Corollary 4.8)

Theorem For any $d, n \in \mathbb{N}>0$, there exists a lattice $R_{d, n}$ (with $R_{d, n} \simeq R_{d^{\prime}, n^{\prime}}$ if

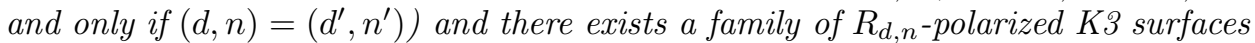
such that, for any $m \in \mathbb{N}_{>0}$ and any $R_{d, n}$-polarized $K 3$ surface $X$ there exists an $R_{d, m}$-polarized $K 3$ surface $Y$ isogenous to $X$ with an isogeny of degree $2^{|n-m|}$.

So for each $d \in \mathbb{N}_{>0}$ there are countably many families of polarized K3 surfaces, such that there exists an isogeny between members of each family.

The Néron-Severi group and the transcendental lattice of all the surfaces involved in these isogenies are explicitly given. In Section 4.3 we describe the Galois closure of the $2^{2}: 1$ (non Galois) covers constructed. Moreover, in Section 4.4 we describe the geometry of a generic member $X_{2}$ of a certain maximal dimensional family of $\mathrm{K} 3$ surfaces which is contained in $\mathcal{L}_{2} \cap \mathcal{M}_{2}$. The K3 surface $X_{2}$ admits two different polarizations of degree 4: one exhibits the surface $X_{2}$ as a special singular quartic in $\mathbb{P}^{3}$ with eight nodes, the other as smooth double cover of a quadric in $\mathbb{P}^{3}$. The former model is the singular quotient of another K3 surface by a symplectic involution (thus it implies that $X_{2} \in \mathcal{M}_{2}$ ), the latter implies that $X_{2}$ admits a symplectic involution induced by the switching of the rulings on the quadric (thus it implies that $X_{2} \in \mathcal{L}_{2}$ ). We describe both projective models of $X_{2}$ and give the explicit relation between them, providing a geometric realization of the previous lattice theoretic result which guarantees that $X_{2}$ is both covered by a K3 surface 
and is a cover of a K3 surface. In particular this allows us to describe a symplectic involution on the model of $X_{2}$ as singular quotient. In Section 4.5 we analyse the similar problem for two specific families of codimension 1 in $\mathcal{L}_{2} \cap \mathcal{M}_{2}$; one of these families is totally contained in all the components of $\mathcal{L}_{2}$ and the other in all the components of $\mathcal{M}_{2}$.

Acknowledgements. Part of this project was realized while the first-named author was visiting the Max Planck Institute of Mathematics in Bonn: the first-named author is grateful to Max Planck Institute for Mathematics in Bonn for its hospitality and financial support. We are grateful to Bert van Geemen for his enlightening suggestions and to Simon Brandhorst for his precious remarks.

\section{Preliminary Results}

We recall in this section some of the definitions and results on K3 surfaces, symplectic automorphisms on K3 surfaces and quotients of K3 surfaces by their automorphisms. In the following we work with projective surfaces.

\subsection{Symplectic automorphisms and cyclic covers of K3 surfaces.}

Definition 2.1. A (projective) K3 surface is a regular projective surface with trivial canonical bundle. If $X$ is a K3 surface, we choose a generator of $H^{2,0}(X)$, (i.e. a symplectic form), we denote it by $\omega_{X}$ and we call it the period of the K3 surface. The second cohomology group $H^{2}(X, \mathbb{Z})$ of a K3 surface $X$ equipped with the cup product is a lattice, isometric to a standard lattice which does not depend on $X$ and is denoted by $\Lambda_{K 3}:=U^{\oplus 3} \oplus E_{8}(-1)^{\oplus 2}$.

Definition 2.2. Let $X$ be a K3 surface, and $\omega_{X}$ its period. An automorphism $\sigma$ of $X$ is said to be symplectic if $\sigma^{*}\left(\omega_{X}\right)=\omega_{X}$.

One of the main results on symplectic automorphisms on K3 surfaces is that the quotient of a K3 surface by a symplectic automorphism is still a K3 surface, after a birational transformation which resolves the singularities of the surface.

Proposition 2.3. ([N2]) Let $X$ be a K3 surface and $\sigma \in A u t(X)$ a finite automorphism of $X$. Then the minimal smooth surface $Y$ birational to $X / \sigma$ is a K3 surface if and only if $\sigma$ is symplectic.

Definition 2.4. We will say that a K3 surface $Y$ is $n: 1$ cyclically covered by a K3 surface, if there exists a pair $(X, \sigma)$ such that $X$ is a K3 surface, $\sigma$ is an automorphism of order $n$ of $X$ and $Y$ is birational to $X / \sigma$.

The first mathematician who worked on symplectic automorphisms of finite order on K3 surfaces and who established the fundamental results on these automorphisms was Nikulin, in [N2. We summarize in Theorem 2.7 and Theorem 2.9 the main results obtained in his paper, but first we recall some useful information and definitions.

If $\sigma$ is a symplectic automorphism on $X$ of order $n$, its linearization near the points with non trivial stabilizer is given by a $2 \times 2$ diagonal matrix with determinant 1 and thus it is of the form $\operatorname{diag}\left(\zeta_{n}^{a}, \zeta_{n}^{n-a}\right)$ for $1 \leq a \leq n-1$ and $\zeta_{n}$ an $n$-th primitive root of unity. So, the points with non trivial stabilizer are isolated fixed points and the quotient $X / \sigma$ has isolated singularities, all of type $A_{m_{j}}$ where $m_{j}+1$ divides $n$. In particular the surface $Y$, which is the minimal surface resolving the singularities 
of $X / \sigma$, contains smooth rational curves $M_{i}$ arising from the desingularization of $X / \sigma$. The classes of these curves span a lattice isometric to $\oplus_{j} A_{m_{j}}$.

Definition 2.5. Let $Y$ be a K3 surface, $n: 1$ cyclically covered by a K3 surface. The minimal primitive sublattice of $\mathrm{NS}(Y)$ containing the classes of the curves $M_{i}$, arising from the desingularization of $X / \sigma$, is denoted by $\mathbb{M}_{n}$.

We observe that $\mathbb{M}_{n}$ is necessarily an overlattice of finite index (a priori possibly 1) of the lattice $\oplus_{j} A_{m_{j}}$ spanned by the curves $M_{i}$. The presence of a smooth cyclic cover of $X / \sigma$ branched over the singular points obtained as contraction of the curves $M_{i}$ suggests that there are some divisibility relations among the $M_{i}$ 's and thus that the index of the inclusion $\left\langle\left(M_{i}\right)_{i}\right\rangle \hookrightarrow \mathbb{M}_{n}$ would not be 1 (as indeed stated in Theorem 2.77).

Definition 2.6. (See [N2, Definition 4.6]) Let $\sigma$ be an order $n$ automorphism of a K3 surface $X$. We will say that its action on the second cohomology group is essentially unique if there exists an isometry $g_{n}: \Lambda_{K 3} \stackrel{\sim}{\longrightarrow} \Lambda_{K 3}$ of order $n$ of $\Lambda_{K 3}$ such that for every pair $(X, \sigma)$, there exists an isometry $\varphi: H^{2}(X, \mathbb{Z}) \rightarrow \Lambda_{K 3}$ such that $\sigma^{*}=\varphi^{-1} \circ g_{n} \circ \varphi$.

Theorem 2.7. Let $X$ be a $K 3$ surface and $\sigma$ a finite symplectic automorphism of $X$ of order $|\sigma|=n$. Then

- $2 \leq n \leq 8$ (see [N2, Theorem 6.3]);

- the singularities of $X / \sigma$ depend only on $n$ (see [N2, Section 5]);

- the class of isometry of the lattice $\mathbb{M}_{n}$ depends only on $n$ and $\mathbb{M}_{n}$ is an overlattice of index $n$ of the lattice $\left\langle\left(M_{i}\right)_{i}\right\rangle$ spanned by the curves arising from the desingularization of the quotient $X / \sigma$ (see [N2, Theorem 6.3]);

- the action of $\sigma^{*}$ on $H^{2}(X, \mathbb{Z})$ is essentially unique (see [N2, Theorem 4.7]) and thus the classes of isometry of the lattices $H^{2}(X, \mathbb{Z})^{\sigma^{*}}$ and $\left(H^{2}(X, \mathbb{Z})^{\sigma^{*}}\right)^{\perp}$ depend only on $n$.

- The lattice $\left(H^{2}(X, \mathbb{Z})^{\sigma}\right)^{\perp}$ is primitively embedded in $\mathrm{NS}(X)$ (see $\mathbb{N} 2$, Lemma 4.2]) and $\operatorname{rank}\left(\left(\Lambda_{K 3}^{g_{n}}\right)^{\perp}\right)=\operatorname{rank}\left(\mathbb{M}_{n}\right)$ (see [N2, Formula (8.12)]).

Definition 2.8. Let $X$ be a K3 surface with a symplectic automorphism $\sigma$ of order $n$. Since the action of $\sigma^{*}$ on $H^{2}(X, \mathbb{Z})$ is essentially unique, the lattice $\left(H^{2}(X, \mathbb{Z})^{\sigma^{*}}\right)^{\perp}$ is isometric to $\left(\Lambda_{K 3}^{g_{n}}\right)^{\perp}$ (with the notation of Definition [2.6) and we denote it by $\Omega_{n}$.

For every admissible $n$ the lattices $\Omega_{n}$ were computed: in vGS] and Mo if $n=2$; in GSar2 if $n$ is an odd prime; in GSar3 if $n$ is not a prime.

The lattices $\mathbb{M}_{n}$ were computed for every admissible $n$ in $\mathbb{N} 2$, Theorems 6.3 and 7.1].

The lattices $\Omega_{n}$ and $\mathbb{M}_{n}$ characterize the K3 surfaces admitting a symplectic automorphism of order $n$ or a $n: 1$ cyclic cover by a K3 surface respectively; indeed, the following two results hold

Theorem 2.9. (See [N2, Theorem 4.15]) A K3 surface $X$ admits a symplectic automorphism of order $n$ if and only if $\Omega_{n}$ is primitively embedded in $\operatorname{NS}(X)$.

Theorem 2.10. (See [GSar1, Proposition 2.3] for the case $n=2$ and [G2, Theorem 5.2] for other $n)$ A K3 surface $Y$ is $n: 1$ cyclically covered by a K3 surface if and only if $\mathbb{M}_{n}$ is primitively embedded in $\mathrm{NS}(Y)$. 
Corollary 2.11. Let $X$ be a projective $K 3$ surface admitting a symplectic automorphism of order $n$. Then $\rho(X) \geq 1+\operatorname{rank}\left(\Omega_{n}\right)$ and if $\rho(X)=1+\operatorname{rank}\left(\Omega_{n}\right)$, then $\mathrm{NS}(X)$ is an overlattice of finite index (possibly 1) of $\langle 2 d\rangle \oplus \Omega_{n}$, for a certain $d \in \mathbb{N}_{>0}$, such that $\Omega_{n}$ is primitively embedded in this overlattice.

Let $Y$ be a projective $K 3$ surface $n: 1$ cyclically cover by a K3 surface. Then $\rho(Y) \geq 1+\operatorname{rank}\left(\mathbb{M}_{n}\right)$ and if $\rho(Y)=1+\operatorname{rank}\left(\mathbb{M}_{n}\right)$, then $\mathrm{NS}(Y)$ is an overlattice of finite index (possibly 1) of $\langle 2 e\rangle \oplus \mathbb{M}_{n}$, for a certain $e \in \mathbb{N}_{>0}$, such that $\mathbb{M}_{n}$ is primitively embedded in this overlattice.

Proof. Since $X$ admits a symplectic automorphism of order $n, \Omega_{n}$ is primitively embedded in $\operatorname{NS}(X)$. Since $\Omega_{n}$ is negative definite and $X$ is projective, the orthogonal to $\Omega_{n}$ in $\mathrm{NS}(X)$ contains a class with a positive self intersection, in particular it is non empty. So $\rho(X) \geq 1+\operatorname{rank}\left(\Omega_{n}\right)$ and $\langle 2 d\rangle \oplus \Omega_{n}$ is embedded in $\operatorname{NS}(X)$. Similarly one obtains the result for $\rho(Y)$ and $\mathrm{NS}(Y)$.

Definition 2.12. We define the following sets of K3 surfaces (which are subsets of the moduli space of the K3 surfaces):

$\mathcal{L}_{n}:=\{$ K3 surfaces which admit a symplectic automorphims $\sigma$ of order $n\} / \cong$,

$\mathcal{M}_{n}:=\{K 3$ surfaces which admit an $n: 1$ cyclic cover by a K3 surface $\} / \cong$, where $\cong$ denotes the equivalence relation given by isomorphism between two K3 surfaces.

Given an even hyperbolic lattice $R$ which admits a primitive embedding in $\Lambda_{K 3}$, we denote by $\mathcal{P}(R)$ the moduli space of isomorphism classes of $R$-polarized $K 3$ surfaces, i.e. of those $K 3$ surfaces $X$ for which there exists a primitive embedding $R \subset \mathrm{NS}(X)$. Moreover, we will write $A<B$ in order to say that $B$ is an overlattice of finite index of $A$.

Corollary 2.13. The set $\mathcal{L}_{n}$ is a union of countably many components and each of them is a family of $R$-polarized K3 surfaces, for an appropriate choice of the lattice $R$ :

$$
\mathcal{L}_{n}=\bigcup_{d \in \mathbb{N}}\left(\bigcup_{\substack{\left(\langle 2 d\rangle \oplus \Omega_{n}\right)<R \\ \Omega_{n} \subset R \text { prim. }}} \mathcal{P}(R)\right) .
$$

All the components $\mathcal{P}(R)$ are equidimensional and have dimension $19-\operatorname{rank}\left(\Omega_{n}\right)$.

The set $\mathcal{M}_{n}$ is a union of countably many components and each of them is a family of R-polarized K3 surfaces, for an appropriate choice of the lattice $R$ :

$$
\mathcal{M}_{n}=\bigcup_{d \in \mathbb{N}}\left(\bigcup_{\substack{\left(\langle 2 d\rangle \oplus \mathbb{M}_{n}\right)<R \\ \mathbb{M}_{n} \subset R \text { prim. }}} \mathcal{P}(R)\right) .
$$

All the components are equidimensional and have dimension $19-\operatorname{rank}\left(\mathbb{M}_{n}\right)=$ $19-\operatorname{rank}\left(\Omega_{n}\right)$.

Proof. Let $R$ be an overlattice of finite index of $\langle 2 d\rangle \oplus \Omega_{n}$ such that $\Omega_{n}$ is primitively embedded in it. If $X$ is a K3 surface such that $R$ is primitively embedded in $\operatorname{NS}(X)$, then $\Omega_{n}$ is primitively embedded in $\operatorname{NS}(X)$ and thus $X$ admits a symplectic automorphism of order $n$, by Theorem 2.9. Vice versa, if a projective K3 surface 
$X$ admits a symplectic automorphism of order $n$, then there exists a $d \in \mathbb{N}>0$ such that $\langle 2 d\rangle \oplus \Omega_{n}$ is embedded in $\operatorname{NS}(X)$, and an overlattice $R$ of $\langle 2 d\rangle \oplus \Omega_{n}$ is primitively embedded in $\operatorname{NS}(X)$. So one can describe the set $\mathcal{L}_{n}$ as union of families $\mathcal{P}(R)$ of $R$-polarized K3 surfaces, where $R$ is a proper overlattice of index $r$ (possibly 1 ) of $\langle 2 d\rangle \oplus \Omega_{n}$ for a certain $d \in \mathbb{N}$. There are countably many lattices $\langle 2 d\rangle \oplus \Omega_{n}$ and each of them has a finite number of overlattices of finite index. So $\mathcal{L}_{n}$ is the union of countably many families of $R$-polarized K3 surfaces. The dimension of each of these families is $20-\operatorname{rank}(R)=20-\left(1+\operatorname{rank}\left(\Omega_{n}\right)\right)$. This concludes the proof for the set $\mathcal{L}_{n}$.

The proof for $\mathcal{M}_{n}$ is similar, but one has to use the Theorem 2.10 instead of the Theorem 2.9.

2.2. Isogenies between $\mathbf{K} 3$ surfaces. The following definition was first given by Inose in [I] in the case of K3 surfaces with Picard number 20.

Definition 2.14. Let $X$ and $Y$ be two $K 3$ surfaces. We say that $X$ and $Y$ are isogenous if there exists a rational map of finite degree between $X$ and $Y$. This map is said to be an isogeny between $X$ and $Y$ and if it is generically of degree $n$, the map is said to be an isogeny of degree $n$.

The easiest construction of an isogeny between K3 surfaces is given by the quotient by a finite symplectic automorphism, i.e. if $X$ is a $\mathrm{K} 3$ surface admitting a symplectic automorphism $\sigma$ of order $n$, then the quotient map induces an isogeny of degree $n$ between $X$ and $Y$, the minimal model of $X / \sigma$. So if $X \in \mathcal{L}_{n}$, then there exists $Y \in \mathcal{M}_{n}$ which is isogenous to $X$ with an isogeny of degree $n$. Similarly if $Y \in \mathcal{M}_{n}$, then there exists a K3 surface $X \in \mathcal{L}_{n}$ which is isogenous to $Y$ with an isogeny $X \rightarrow Y$ of degree $n$.

There exist however isogenies between K3 surfaces which are not induced by the quotient by a finite group of symplectic automorphisms: an example is given by isogenous Kummer surfaces constructed from Abelian surfaces related by an isogeny, as in [I, Proof of Thm 2], under the additional assumption that the degree is a prime $p>7$, (see also [BSV, Example 6.5]).

Now, let us suppose that $Z$ is a K3 surface such that $Z \in \mathcal{L}_{n} \cap \mathcal{M}_{n}$. Then, there exists a K3 surface $X \in \mathcal{L}_{n}$ which is isogenous to $Z$, with an isogeny $\rho: X \rightarrow Z$ of degree $n$, but also a $\mathrm{K} 3$ surface $Y \in \mathcal{M}_{n}$ which is isogenous to $Z$ with an isogeny $\pi: Z \rightarrow Y$ of degree $n$. So the existence of $Z \in \mathcal{L}_{n} \cap \mathcal{M}_{n}$ allows one to construct an isogeny of degree $n^{2}$ between the two K3 surfaces $X$ and $Y$, given by the composition $\pi \circ \rho: X \rightarrow Y$. We will show that in many cases this isogeny is not induced by a quotient by a finite group of symplectic automorphisms acting on $X$, see Proposition 3.11 .

In the Section 3 we prove that $\mathcal{L}_{n} \cap \mathcal{M}_{n}$ is non empty if $2 \leq n \leq 8$ and then we provide examples of $n^{2}: 1$ isogenies between K3 surfaces.

2.3. Remarks on Hodge isogenies between K3 surfaces. The Definition 2.14 is not the only notion of isogeny existing in the literature: to distinguish between the two definitions, we will talk here of Hodge isogeny for the notion used for example in $[\mathrm{Bu}, \mathrm{Huy}$.

Definition 2.15. Let $X$ and $Y$ be two $K 3$ surfaces. We say the $X$ and $Y$ are Hodge isogenous if there exists a rational Hodge isometry between $H^{2}(X, \mathbb{Q})$ and $H^{2}(Y, \mathbb{Q})$. 
Hodge isogenous K3 surfaces have been studied since foundational work of [M] and [N3, also in relation with Šafarevič's conjecture [Sa] about algebraicity of correspondences on $K 3$ surfaces.

In BSV, Prop. 3.1], the authors give a comparison between the notion of isogeny and of Hodge isogeny:

Proposition 2.16. If $\varphi: X \rightarrow Y$ is an isogeny of order $n, n$ is not a square and the rank of the transcendental lattices $T_{X}$ and $T_{Y}$ is odd, $\varphi$ is never a Hodge isogeny.

This follows from the fact that, under these assumptions, there cannot exist any isometry $T_{X} \otimes \mathbb{Q} \simeq T_{Y} \otimes \mathbb{Q}$. The transcendental lattice $T_{X}$ of the very general K3 surface $X \in \mathcal{L}_{n}$ has always odd rank (see Theorem [3.9); by Proposition 2.16] if $n$ is not a square, so if $n \neq 4$, the surface $X$ is never Hodge isogenous to the minimal resolution of its quotient. The assumption on the degree $n$ is in particular due to the following straightforward fact:

Lemma 2.17. For any non degenerate lattice $T$ and any integer $n \in \mathbb{N}$, there exists an isometry $T \otimes \mathbb{Q} \simeq T\left(n^{2}\right) \otimes \mathbb{Q}$.

Proposition 2.18. For any $n \in \mathbb{N}$, if $\varphi: X \rightarrow Y$ is an isogeny of degree $n^{2}$, then $X$ and $Y$ are Hodge isogenous.

Proof. It is proven in BSV, Proposition 3.2] that $T_{X} \otimes \mathbb{Q} \simeq T_{Y} \otimes \mathbb{Q}$ if and only if $T_{Y} \otimes \mathbb{Q} \simeq T_{Y}\left(n^{2}\right) \otimes \mathbb{Q}$, which is true by Lemma 2.17. Then Witt's theorem implies that the isometry $T_{X} \otimes \mathbb{Q} \simeq T_{Y} \otimes \mathbb{Q}$ extends to a Hodge isometry $H^{2}(X, \mathbb{Q}) \simeq$ $H^{2}(Y, \mathbb{Q})$.

In Proposition 3.11 we construct isogenies of degree $n^{2}$ between K3 surfaces; Proposition 2.18 implies that they are necessarily Hodge isogenies.

One of the interesting properties of Hodge isogenous K3 surfaces is that they have isomorphic rational motives, by [Huy, Theorem 0.2]. This also holds in the case described above of a K3 surface $X$ isogenous to the minimal model $Y$ of the quotient $X / \sigma$, as shown for example in $[\mathrm{L}$, Proof of Thm 3.1] following the argument of $[\mathrm{P}$, but to the knowledge of the authors it is still an open question for a general isogeny.

\section{The intersection $\mathcal{L}_{n} \cap \mathcal{M}_{n}$}

The main result in this section is Theorem 3.9, where we exhibit the maximal dimensional components of $\mathcal{L}_{n} \cap \mathcal{M}_{n}$. As preliminary result, we describe in 3.1 a specific family of K3 surfaces contained in $\mathcal{L}_{n} \cap \mathcal{M}_{n}$. This family is related with a special isogeny between K3 surfaces, which is induced by an isogeny between elliptic curves, see Remark 3.3 .

3.1. The $\left(U \oplus \mathbb{M}_{n}\right)$-polarized $\mathbf{K 3}$ surfaces. The $\left(U \oplus \mathbb{M}_{n}\right)$-polarized $\mathrm{K} 3$ surfaces have interesting geometric properties: this family is considered for $n=2$ in $\mathrm{vGS}$, and for other values of $n$ in GSar2 and GSar3 to find explicitly $\Omega_{n}$. Here we reconsider it as example of a family of K3 surfaces contained in $\mathcal{L}_{n} \cap \mathcal{M}_{n}$.

Proposition 3.1. Let $2 \leq n \leq 8$ and $\mathcal{U}_{n}:=\mathcal{P}\left(U \oplus \mathbb{M}_{n}\right)$ be the family of the $\left(U \oplus \mathbb{M}_{n}\right)$-polarized K3 surfaces. Then:

- $\mathcal{U}_{n}$ is non empty and has dimension $18-\operatorname{rank}\left(\mathbb{M}_{n}\right)$; 
- if $S$ is a K3 surface such that $S \in \mathcal{U}_{n}$, then $S$ admits an elliptic fibration $\mathcal{E}_{n}: S \rightarrow \mathbb{P}^{1}$ with an $n$-torsion section $t$;

- $\mathcal{U}_{n} \subset \mathcal{L}_{n} \cap \mathcal{M}_{n}$

- if $S \in \mathcal{U}_{n}$ and $\sigma_{t}$ is the translation by $t$ on $\mathcal{E}_{n}$, the minimal model of $S / \sigma_{t}$ is a K3 surface in $\mathcal{U}_{n}$.

Proof. The family $\mathcal{U}_{n}$ is non empty for each $n$ such that $2 \leq n \leq 8$ as showed for example in [Sh, Table 2] or [G1, Table 1]. The dimension of $\mathcal{U}_{n}$ follows directly by the fact that the dimension of a non-empty family $\mathcal{P}(R)$ of $R$-polarized K3 surfaces (for a certain lattice $R$ ) is $20-\operatorname{rank}(R)$, and in this case $R \simeq U \oplus \mathbb{M}_{n}$ has rank $2+\operatorname{rank}\left(\mathbb{M}_{n}\right)$. The family $\mathcal{U}_{n}$ was considered in [G1, Proposition 4.3], where it is proved that the set of K3 surfaces admitting an elliptic fibration with a torsion section of order $n$ coincides with the set of $\left(U \oplus \mathbb{M}_{n}\right)$-polarized K3 surfaces. Since $\mathbb{M}_{n}$ is clearly primitively embedded in $U \oplus \mathbb{M}_{n}$, all the K3 surfaces in $\mathcal{U}_{n}$ are also contained in $\mathcal{M}_{n}$. Moreover, let $\mathcal{E}_{n}: S \rightarrow \mathbb{P}^{1}$ be an elliptic fibration on $S$ with an $n$-torsion section $t$. This allows to consider $S$ as an elliptic curve over the field of functions $k\left(\mathbb{P}^{1}\right)$ and the presence of an $n$-torsion section is equivalent to the presence of an $n$-torsion rational point on this elliptic curve. So the translation by $t$ is well defined and it induces an automorphism of order $n$ on $S$. This is a symplectic automorphism (it is the identity on the base of the fibration and acts on the smooth fibers preserving their periods). We denote this symplectic automorphism by $\sigma_{t}$. Since $S \in \mathcal{U}_{n}$ admits a symplectic automorphism of order $n, \mathcal{U}_{n} \subset \mathcal{L}_{n}$ and thus $\mathcal{U}_{n} \subset \mathcal{L}_{n} \cap \mathcal{M}_{n}$. In [G1, Proposition 4.3] it is also proved that the quotient of an elliptic fibration with basis $\mathbb{P}^{1}$ by the translation by a torsion section is another elliptic fibration over $\mathbb{P}^{1}$ with an $n$-torsion section. Thus $S / \sigma_{t}$ admits a smooth minimal model with an elliptic fibration with an $n$-torsion section and this minimal model is a K3 surface (since $\sigma_{t}$ is a symplectic automorphism). Thus the minimal model of $S / \sigma_{t}$ belongs to the family $\mathcal{U}_{n}$.

Corollary 3.2. For every $n$ such that $2 \leq n \leq 8, \mathcal{L}_{n} \cap \mathcal{M}_{n}$ is non empty.

Proof. The intersection $\mathcal{L}_{n} \cap \mathcal{M}_{n}$ contains at least the non empty family $\mathcal{U}_{n}$.

Remark 3.3. Since both $\mathcal{L}_{n}$ and $\mathcal{M}_{n}$ are the union of $\left(19-\operatorname{rank}\left(\mathbb{M}_{n}\right)\right)$-dimensional families of polarized K3 surfaces, the intersection between these sets is at most $\left(19-\operatorname{rank}\left(\mathbb{M}_{n}\right)\right)$-dimensional. The Proposition 3.1 provides an intersection in a codimension 1 subfamily. In the Theorem 3.9 we will see that one can obtain larger intersection.

Remark 3.4. Since each $\left(U \oplus \mathbb{M}_{n}\right)$-polarized K3 surface has an elliptic fibration, it can be interpreted as elliptic curve over the field of rational functions in one variable. The symplectic automorphism which induces the isogeny between the two K3 surfaces in $\mathcal{U}_{n}$ as in Proposition 3.1 is an isogeny of the associated elliptic curve over the field of rational functions.

3.2. Maximal dimensional components of $\mathcal{L}_{n} \cap \mathcal{M}_{n}$. In this section we prove that there are components of $\mathcal{L}_{n}$ completely contained in $\mathcal{M}_{n}$ and vice versa. The proof is lattice theoretic: in order to obtain this result, we need some extra information on the lattices $\mathbb{M}_{n}$ and $\Omega_{n}$. Both these lattices are primitively embedded in the Néron-Severi group of a $\left(U \oplus \mathbb{M}_{n}\right)$-polarized K3 surface, so we now use the K3 surfaces in the family $\mathcal{U}_{n}$ to compare the discriminant forms of $\mathbb{M}_{n}$ and $\Omega_{n}$. 
Proposition 3.5. Let $A_{\Omega_{n}}$ (resp. $A_{\mathbb{M}_{n}}$ ) the discriminant group of $\Omega_{n}$ (resp. $\mathbb{M}_{n}$ ) and $q_{\Omega_{n}}$ (resp. $q_{\mathbb{M}_{n}}$ ) its discriminant form. Then $A_{\Omega_{n}}=(\mathbb{Z} / n \mathbb{Z})^{\oplus 2} \oplus A_{\mathbb{M}_{n}}$ and $q_{\Omega_{n}}=u(n) \oplus q_{\mathbb{M}_{n}}$, where $u(n)$ is the discriminant form of the lattice $U(n)$.

Proof. Nikulin proved that $A_{\Omega_{n}}=(\mathbb{Z} / n \mathbb{Z})^{\oplus 2} \oplus A_{\mathbb{M}_{n}}$ in [N2, Lemma 10.2]. By the Proposition 3.1, if $\mathrm{NS}(S) \simeq U \oplus \mathbb{M}_{n}$, then $S$ admits an elliptic fibration $\mathcal{E}_{n}: S \rightarrow \mathbb{P}^{1}$ with an $n$-torsion section $t$ and thus a symplectic automorphism $\sigma_{t}$, which is the translation by $t$. Let us denote by $F$ the class in $\mathrm{NS}(S)$ of the fiber of the elliptic fibration $\mathcal{E}_{n}$, by $O$ the class of the zero section, by $t$ the class of the $n$-torsion section, by $t_{i}, i=2, \ldots, n-1$, the class of the section corresponding to the sum of $t$ with itself $i$ times in the Mordell-Weil group. By definition $\sigma_{t}$ preserves the classes $F$ and $O+t+\sum_{i=2}^{n-1} t_{i}$. So $U(n) \simeq\left\langle F, O+t+\sum_{i=2}^{n-1} t_{i}\right\rangle \subset \mathrm{NS}(S)^{\sigma_{t}}$ and thus $\left\langle F, O+t+\sum_{i=2}^{n-1} t_{i}\right\rangle^{\perp} \supset\left(\operatorname{NS}(S)^{\sigma_{t}}\right)^{\perp}$. Since $\operatorname{rank}\left(\Omega_{n}\right)=\operatorname{rank}\left(\mathbb{M}_{n}\right), \operatorname{rank}\left(\Omega_{n}\right)=$ $\rho(S)-2$. So

$$
\left\langle F, O+t+\sum_{i=2}^{n-1} t_{i}\right\rangle^{\perp} \supset\left(\mathrm{NS}(S)^{\sigma_{t}}\right)^{\perp} \simeq \Omega_{n} .
$$

Denoted by $T_{S}$ the transcendental lattice of $S$, it follows from $\mathrm{NS}(S) \simeq U \oplus \mathbb{M}_{n}$ that $q_{T_{S}}=-q_{\mathbb{M}_{n}}$. By $\left(\mathrm{NS}(S)^{\sigma_{t}}\right)^{\perp} \simeq \Omega_{n}$ one obtains that the orthogonal of $\Omega_{n}$ in $H^{2}(S, \mathbb{Z})$ is an overlattice of finite index (possibly 1) of $U(n) \oplus T_{S}$. Since $A_{\Omega_{n}}=(\mathbb{Z} / n \mathbb{Z})^{2} \oplus A_{\mathbb{M}_{n}}$, the orthogonal of $\Omega_{n}$ in $H^{2}(S, \mathbb{Z})$ is $U(n) \oplus T_{S}$. So $q_{\Omega_{n}} \simeq-q_{U(n) \oplus T_{S}}=u(n) \oplus q_{\mathbb{M}_{n}}$.

Lemma 3.6. Let $F$ be a finite abelian group with quadratic form $q_{F}$ and $m \geq 2$. Let $V=\langle 2 d\rangle \oplus W$ be an indefinite even non-degenerate lattice with discriminant group $A_{V}=(\mathbb{Z} / 2 d \mathbb{Z}) \oplus(\mathbb{Z} / m \mathbb{Z})^{\oplus 2} \oplus F$, with discriminant form $q_{A_{V}}=\left(\frac{1}{2 d}\right) \oplus u(m) \oplus q_{F}$. If $d \equiv 0 \bmod 2 m$, then $V$ admits an overlattice $Z$ of index $m$ with $A_{Z}=\mathbb{Z} / 2 d \mathbb{Z} \oplus F$ and $q_{A_{Z}}=\left(\frac{1}{2 d}\right) \oplus q_{F}$. Moreover, $Z$ contains primitively $W$.

Proof. By assumption, there exists some integer $k$ such that $2 d=4 \mathrm{~km}$. Let $h$ be a generator of the $\mathbb{Z} / 2 d \mathbb{Z}$ summand of $A_{V}$ such that $h^{2}=\frac{1}{2 d}$, and let $e_{1}, e_{2}$ be a basis of the $(\mathbb{Z} / m \mathbb{Z})^{\oplus 2}$ summand in $A_{V}$ such that $e_{1}^{2}=e_{2}^{2}=0$ and $e_{1} e_{2}=-\frac{1}{m}$. We define $\epsilon:=e_{1}+2 k e_{2}$, so that $\epsilon^{2}=-\frac{4 k}{m}$. Then the subgroup $H:=\langle(4 k) h+\epsilon\rangle$ is isotropic and its orthogonal inside $A_{V}$ is $H^{\perp}=\left\langle h-e_{2}, \nu\right\rangle \oplus F$, where $\nu:=e_{1}-2 k e_{2}$. It follows from [N1, Propostion 1.4.1 and Corollary 1.10.2] that there exists an even overlattice $Z$ of $V$ of index $m$ with $A_{Z} \cong H^{\perp} / H=\left\langle h-e_{2}\right\rangle \oplus F \cong \mathbb{Z} / 2 d \mathbb{Z} \oplus F$, and $q_{A_{Z}}$ is induced on the quotient $H^{\perp} / H$ by $\left(q_{A_{V}}\right)_{\mid H^{\perp}}$, so it is exactly $\left(\frac{1}{2 d}\right) \oplus q_{F}$. Finally, we observe that the intersection of $H$ with $A_{W}$ inside $A_{V}$ is trivial, hence $W$ is a primitive sublattice of $Z$.

Corollary 3.7. Let $2 \leq n \leq 8, d \in \mathbb{N}, d \geq 1$ and $d \equiv 0 \bmod 2 n$. Then $\langle 2 d\rangle \oplus$ $\Omega_{n}$ admits an overlattice of index $n$ whose discriminant form is $\left(\frac{1}{2 d}\right) \oplus q_{\mathbb{M}_{n}}$; this overlattice contains primitively $\Omega_{n}$.

Proof. It suffices to apply Lemma 3.6 to the lattice $V=\langle 2 d\rangle \oplus \Omega_{n}$ and to recall that $q_{\Omega_{n}}=u(n) \oplus q_{\mathbb{M}_{n}}$, by Proposition 3.5 .

Definition 3.8. For each $2 \leq n \leq 8$ and each $d \in \mathbb{N}, d \geq 1$, we denote by $L_{d, n}$ the lattice $\langle 2 d\rangle \oplus \Omega_{n}$.

For each $2 \leq n \leq 8$ and each $d \in \mathbb{N}, d \geq 1$, and $d \equiv 0 \bmod 2 n$, we denote by $L_{d, n}^{\prime}$ the overlattice of index $n$ of $L_{d, n}$ constructed in Corollary 3.7 . 
For each $2 \leq n \leq 8$ and each $e \in \mathbb{N}, e \geq 1$, we denote by $M_{e, n}$ the lattice $\langle 2 e\rangle \oplus \mathbb{M}_{n}$.

Theorem 3.9. Let $d \in \mathbb{N}, d \geq 1$ and $d \equiv 0 \bmod 2 n$. The lattice $L_{d, n}^{\prime}$ is unique in its genus and

$$
L_{d, n}^{\prime} \simeq M_{d, n}
$$

The family $\mathcal{P}\left(L_{d, n}^{\prime}\right)$ is $\left(19-\operatorname{rank}\left(\Omega_{n}\right)\right)$-dimensional and is a subset of $\mathcal{L}_{n} \cap \mathcal{M}_{n}$, i.e. each $K 3$ surface in this family admits a symplectic automorphism of order $n$ and is $n: 1$ cyclically covered by a K3 surface.

Proof. By the Corollary 3.7 the lattice $L_{d, n}^{\prime}$ has the same discriminant group and form as the lattice $M_{d, n}$. By [N2, Proposition 7.1], the length and the rank of the lattice $\mathbb{M}_{n}$ are the following:

\begin{tabular}{|c|c|c|c|c|c|c|c|}
\hline$n$ & 2 & 3 & 4 & 5 & 6 & 7 & 8 \\
\hline$l\left(\mathbb{M}_{n}\right)$ & 6 & 4 & 4 & 2 & 2 & 1 & 2 \\
\hline $\operatorname{rank}\left(\mathbb{M}_{n}\right)$ & 8 & 12 & 14 & 16 & 16 & 18 & 18 \\
\hline
\end{tabular}

where the length $l(R)$ of a lattice $R$ is the minimal number of generators of the discriminant group $R^{\vee} / R$. Since $\operatorname{rank}\left(M_{d, n}\right)=1+\operatorname{rank}\left(\mathbb{M}_{n}\right)$ and, if $d \equiv 0 \bmod 2 n$, $l\left(\langle 2 d\rangle \oplus \mathbb{M}_{n}\right)=1+l\left(\mathbb{M}_{n}\right)$, for every admissible $n$ and $d \equiv 0 \bmod 2 n, \operatorname{rank}\left(M_{d, n}\right) \geq$ $2+l\left(M_{d, n}\right)$, so by [N1, Corollary 1.13.3], there is a unique even hyperbolic lattice with the same rank, length, discriminant group and form as $M_{d, n}$. Since $L_{d, n}^{\prime}$ has all the prescribed properties, we conclude that $L_{d, n}^{\prime} \simeq M_{d, n}$. Moreover, by [N1, Theorem 1.14.4], if $n<7$ the lattice $L_{d, n}^{\prime} \simeq M_{d, n}$ admits a unique, up to isometry, primitive embedding in $\Lambda_{K 3}$, and thus determines a $\left(19-\operatorname{rank}\left(\Omega_{n}\right)\right)$-dimensional family of K3 surfaces. If $n=7,8$, any primitive embedding of $L_{d, n}^{\prime} \simeq M_{d, n}$ in the unimodular lattice $\Lambda_{K 3}$, which exists by results in GSar2, GSar3, identifies the same genus of the orthogonal complement $T_{d, n}$ of rank three and signature $(2,1)$ : we get respectively that $A_{T_{d, 7}}=\mathbb{Z} / 7 \mathbb{Z} \oplus \mathbb{Z} / 2 d \mathbb{Z}$ and $A_{T_{d, 8}}=\mathbb{Z} / 2 \mathbb{Z} \oplus Z / 4 \mathbb{Z} \oplus \mathbb{Z} / 2 d \mathbb{Z}$ with quadratic forms $q_{T_{d, 7}}=\left(-\frac{4}{7}\right) \oplus\left(-\frac{1}{2 d}\right)$ and $q_{T_{d, 8}}=\left(\frac{1}{2}\right) \oplus\left(\frac{1}{4}\right) \oplus\left(-\frac{1}{2 d}\right)$. It follows from [N1, Proposition 1.15.1] that the primitive embedding of $L_{d, n}^{\prime} \simeq M_{d, n}$ in $\Lambda_{K 3}$ is unique, up to isometry, if and only if $T_{d, n}$ is unique in its genus and the map $O\left(T_{d, n}\right) \rightarrow O\left(q_{T_{d, n}}\right)$ is surjective. By [MM, Theorem VIII.7.5], these two conditions hold in particular if the discriminant quadratic form $q_{T_{d, n}}$ is $p$-regular for all prime numbers $p \neq s$ and it is $s$-semiregular for a single prime number $s$. The precise (and quite technical) definition of $p$-regular and $p$-semiregular form can be found in [MM, Definition VIII.7.4]. An easy application of [MM, Lemma VIII.7.6 and VIII.7.7] implies that:

- $q_{T_{d, 7}}$ is $p$-regular if $p \neq 7$ and it is 7-semiregular;

- $q_{T_{d, 8}}$ is $p$-regular if $p \neq 2$ and it is 2 -semiregular.

Hence, also for $n=7,8, T_{d, n}$ is unique in its genus and the primitive embedding of $L_{d, n}^{\prime} \simeq M_{d, n}$ in $\Lambda_{K 3}$ is unique up to isometry, and thus determines a $\left(19-\operatorname{rank}\left(\Omega_{n}\right)\right)$ dimensional family of K3 surfaces.

Each K3 surface which is $M_{d, n}$-polarized is contained in $\mathcal{L}_{n} \cap \mathcal{M}_{n}$ because there are primitive embeddings both of $\Omega_{n}$ and of $\mathbb{M}_{n}$ in its Néron-Severi group.

Proposition 3.10. Let $2 \leq n \leq 8$ and $d \in \mathbb{N}, d \geq 1$. The lattice $L_{d, n}$ is not isometric to any overlattice of finite index (possibly 1) of $M_{e, n}$, for any e. In particular if $X$ is a K3 surface such that $\mathrm{NS}(X) \simeq L_{d, n}$, then $X$ does not admit 
a cyclic $n: 1$ cover by a K3 surface and the families of the $\left(L_{d, n}\right)$-polarized $K 3$ surfaces are not (totally) contained in $\mathcal{M}_{n}$.

Proof. By Proposition 3.5, $l\left(\Omega_{n}\right)=2+l\left(\mathbb{M}_{n}\right)$. Hence $l\left(L_{d, n}\right) \geq l\left(\Omega_{n}\right)=2+l\left(\mathbb{M}_{n}\right)>$ $l\left(M_{e, n}\right)$. Since any overlattice of $M_{e, n}$ has at most the length of $M_{e, n}$, the lattices $L_{d, n}$ can not be isometric to any overlattice of the lattice $M_{e, n}$.

In conclusion we proved that there are components of $\mathcal{L}_{n}$ (and of $\mathcal{M}_{n}$ ) which are completely contained in $\mathcal{L}_{n} \cap \mathcal{M}_{n}$, but there are also components of $\mathcal{L}_{n}$, which are not contained in $\mathcal{M}_{n}$, and thus in $\mathcal{L}_{n} \cap \mathcal{M}_{n}$. It is also true that there are components of $\mathcal{M}_{n}$ which are not totally contained in $\mathcal{L}_{n}$ (see e.g. Theorem 4.6 for the case $n=2$.)

In the following proposition we construct an $n^{2}: 1$ isogeny between two K3 surfaces by using a third K3 surface, which is $L_{d, n}^{\prime}$-polarized, and we prove that generically this $n^{2}: 1$ isogeny is not just the quotient by an automorphism group.

Proposition 3.11. Let $Z$ be a $K 3$ surface, such that $\mathrm{NS}(Z)=L_{d, n}^{\prime}$, let $X$ be the K3 surface which is a $n: 1$ cyclic cover of $Z$ and $Y$ be the quotient of $Z$ by a symplectic automorphism of order $n$. Then there is an $n^{2}: 1$ isogeny between $X$ and $Y$ but there is no finite group $G$ of automorphisms on $X$ such that $Y$ is birational to $X / G$.

Proof. By Theorem 3.9 the K3 surfaces $Z$ which are $L_{d, n}^{\prime}$-polarized are $n: 1$ isogenous to two K3 surfaces, $X$ and $Y$, respectively with the two $n: 1$ isogenies $X \rightarrow Z$ and $Z \rightarrow Y$. The composition of these two isogenies is an $n^{2}: 1$ isogeny $X \rightarrow Y$.

If there exists a group of automorphism $G$ as required, it has to be a group of symplectic automorphisms (otherwise the quotient $X / G$ would not be birational to a K3 surface). So $X$ should admit a group of symplectic automorphisms of order $n^{2}$. If $X$ admits a group $G$ of symplectic automorphisms, then $\left(\operatorname{NS}(X)^{G}\right)^{\perp}$ is a lattice (analogous to $\Omega_{n}$ ) which is unique in most of the cases. Its rank depends only on $G$ and it is known for every admissible $G$, see $[\mathrm{H}$. In particular, if $2 \leq n \leq 8$, for every group $G_{n^{2}}$ of order $n^{2}$ acting symplectically on a K3 surface, the rank of the lattice is $\operatorname{rank}\left(\left(\mathrm{NS}(X)^{G_{n^{2}}}\right)^{\perp}\right)>\operatorname{rank}\left(\Omega_{n}\right)$. Hence, if a K3 surface $X$ admits $G_{n^{2}}$ as group of symplectic automorphisms, $\rho(X)>1+\Omega_{n}=\rho(Z)$. But $X$ and $Z$ are isogenous, hence $\rho(X)=\rho(Z)$ and thus $X$ can not admit a group of symplectic automorphisms of order $n^{2}$.

Remark 3.12. In Proposition 3.11 we proved that the $n^{2}: 1$ isogeny $X \rightarrow Y$ is not induced by a quotient map, so that the rational map $X \rightarrow Y$ is not a Galois cover. Let us denote by $V$ its Galois closure, hence $V$ is a surface such that both $X$ and $Y$ are birational to Galois quotients of $V$. Denoted by $G$ the Galois group of the cover $V \rightarrow Y$ and by $H$ the subgroup of $G$ which is the Galois group of $V \rightarrow X, H$ is not a normal subgroup of $G$, otherwise the rational map $X \rightarrow Y$ would be a Galois cover with Galois group $G / H$. The Kodaira dimension of the surface $V$ is non negative (since $V$ covers K3 surfaces), but moreover $V$ can not be a K3 surface. This can be proved applying the same argument of the proof of Proposition 3.11, if $V$ were K3 surface, it would admit $G$ as group of symplectic automorphism, but its Picard number is not big enough. We give more details on the construction of $V$ and $G$ in the case $n=2$, see Section 4.3 


\section{InVOLUTIONS}

In this section we restrict our attention to the case of the symplectic involutions (i.e. $n=2$ ). In this case several more precise and deep results are known about the relations between K3 surfaces admitting a symplectic involutions and K3 surfaces which are their quotients, hence we can improve the general results of the previous section and we can describe explicit examples. In particular we obtain: a complete description of the maximal dimensional components of the intersection $\mathcal{L}_{2} \cap \mathcal{M}_{2}$, in Theorem 4.6, infinite families of K3 surfaces such that for each K3 surface in a family there is another one in another family which is isogenous to it, in Corollary 4.8, geometric examples in Sections 4.4 and 4.5 .

4.1. Preliminary results on symplectic involutions and Nikulin surfaces. For historical reasons, we refer to the K3 surfaces in $\mathcal{M}_{2}$ (i.e. the K3 surfaces which are cyclically 2:1 covered by a K3 surface) as the Nikulin surfaces and to the lattice $\mathbb{M}_{2}$ as the Nikulin lattice, denoted by $N\left(:=\mathbb{M}_{2}\right)$. So we have

Definition 4.1. A Nikulin surface $Y$ is a K3 surface which is the minimal resolution of the quotient of a K3 surface $X$ by a symplectic involution $\sigma$. The minimal primitive sublattice of $\mathrm{NS}(Y)$ containing the curves arising from the desingularization of $X / \sigma$ is denoted by $N$ and it is called Nikulin lattice.

If $\sigma$ is a symplectic involution on a K3 surface $X$, then the fixed locus of $\sigma$ on $X$ consists of 8 isolated points. The quotient surface $X / \sigma$ has 8 singularities of type $A_{1}$, so the minimal resolution $X$ contains 8 disjoint rational curves, which are the exceptional divisors over the singular points of $X / \sigma$. Called $N_{i}, i=1, \ldots, 8$ their classes in the Néron-Severi group, the class $\left(\sum_{i=1}^{8} N_{i}\right) / 2$ is contained in the Néron-Severi group. Indeed the union of the eight disjoint rational curves is the branch locus of the double cover $\widetilde{X} \rightarrow Y$, where $\widetilde{X}$ is the blow up of $X$ in the 8 points fixed by $\sigma$. We will say that a set of rational curves is an even set if the sum of their classes divided by 2 is contained in the Néron-Severi group and that a set of nodes is an even set if the curves resolving these nodes form an even set.

Proposition 4.2. ([N2, Section 6]) The Nikulin lattice is an even negative definite lattice of rank 8 and its discriminant form is the same as the one of $U(2)^{3}$. It contains 16 classes with self-intersection -2 , i.e. $\pm N_{i}, i=1, \ldots, 8$. A $\mathbb{Z}$-basis of $N$ is given by $\left(\sum_{i=1}^{8} N_{i}\right) / 2, N_{i}, i=1, \ldots 7$.

As in the previous section, we will denote by $M_{e, 2}$ the lattice $\langle 2 e\rangle \oplus N$.

Proposition 4.3. (a) A K3 surface $Y$ is a Nikulin surface if and only if the lattice $N$ is primitively embedded in $\mathrm{NS}(Y)$.

(b) The minimal Picard number of a Nikulin surface is 9.

(c) There exists an even overlattice of index two of $M_{e, 2}$ in which $N$ is primitively embedded if and only if $e$ is even. In this case, this lattice is unique up to isometry and denoted by $M_{e, 2}^{\prime}$.

(d) If $Y$ is a Nikulin surface with Picard number 9, then $\mathrm{NS}(Y)$ is isometric either to $M_{e, n}$ or to $M_{e, n}^{\prime}$ for a certain $e$.

Proof. The point $(a)$ is Theorem 2.10, the point $(b)$ is proved in Corollary 2.11 in the case $n=2$. The point $(c)$ is proved in [GSar1, Proposition 2.2] and the point (d) in GSar1, Proposition 2.1]. 
In the case $n=2$, the lattice $\Omega_{2}$ is known to be isometric to $E_{8}(-2)$. As in the previous section, we will denote by $L_{d, 2}$ the lattice $\langle 2 d\rangle \oplus E_{8}(-2)$ and by $L_{d, 2}^{\prime}$ the overlattice of index two of $L_{d, 2}$ such that $L_{d, 2}^{\prime}$ is even and $E_{8}(-2)$ is primitively embedded in $L_{d, 2}^{\prime}$.

Proposition 4.4. (a) A K3 surface $X$ admits a symplectic involution $\sigma$ if and only if the lattice $E_{8}(-2)$ is primitively embedded in $\mathrm{NS}(X)$.

(b) If $X$ admits a symplectic involution, $\rho(X) \geq 9$.

(c) There exists an even overlattice of index two of $L_{d, 2}$ in which $E_{8}(-2)$ is primitively embedded if and only if $d$ is even. In this case, this lattice is unique up to isometry and is $L_{d, 2}^{\prime}$.

(d) If $X$ is a K3 surface admitting a symplectic involution and with Picard number 9, then $\mathrm{NS}(X)$ is isometric either to $L_{d, 2}$ or to $L_{d, 2}^{\prime}$ for a certain d.

Proof. The Proposition follows directly by vGS, Propositions 2.2 and 2.3] (and the points $(a),(b)$ and $(c)$ were already proved in the more general setting of automorphisms of order $n$ in the previous Section).

The main result which is known for involutions and is not yet stated in the more general case of symplectic automorphisms of order $n$, is the explicit relation between the Néron-Severi group of a K3 surface which admits a symplectic involution and the Néron-Severi group of the K3 surface which is its quotient.

Proposition 4.5. (GSar1, Corollary 2.2]) Let $X$ be a K3 surface with a symplectic involution $\sigma$ and $Y$ be the minimal resolution of $X / \sigma$. Then:

- $\mathrm{NS}(X) \simeq L_{e, 2}$ if and only if $\mathrm{NS}(Y) \simeq M_{2 e, 2}^{\prime}$

- $\mathrm{NS}(X) \simeq L_{2 e, 2}^{\prime}$ if and only if $\mathrm{NS}(Y) \simeq M_{e, 2}$.

4.2. The intersection $\mathcal{L}_{2} \cap \mathcal{M}_{2}$ and infinite towers of isogenous $\mathrm{K} 3$ surfaces. Since we know the structure of all the possible Néron-Severi groups of Nikulin surfaces of minimal Picard number (by Proposition 4.3) and all the possible NéronSeveri groups of K3 surfaces of minimal Picard number admitting a symplectic involution (by Proposition 4.4), we are able to give the following refinement of the Theorem 3.9 and of the Proposition 3.10.

Theorem 4.6. A Nikulin surface $Y$ such that $\rho(Y)=9$ admits a symplectic involution if and only if $\mathrm{NS}(Y) \simeq M_{2 d, 2}\left(\simeq L_{2 d, 2}^{\prime}\right)$.

A K3 surface $X$ admitting a symplectic involution such that $\rho(X)=9$ is a Nikulin surface if and only if $\mathrm{NS}(X) \simeq L_{2 d, 2}^{\prime}\left(\simeq M_{2 d, 2}\right)$.

So

$$
\mathcal{L}_{2} \cap \mathcal{M}_{2} \supset \bigcup_{d \in \mathbb{N}>0, d \equiv 0(2)} \mathcal{P}\left(M_{2 d, 2}\right) .
$$

Proof. By Theorem $3.9 M_{2 d, 2} \simeq L_{2 d, 2}^{\prime}$ and thus if $\mathrm{NS}(Y) \simeq M_{2 d, 2}$, then $Y$ admits a symplectic involution. Similarly if $\operatorname{NS}(X) \simeq L_{2 d, 2}^{\prime}, X$ is a Nikulin surface. It remains to prove that if a $\mathrm{K} 3$ surface is in $\mathcal{L}_{2} \cap \mathcal{M}_{2}$, and its Picard number is 9, then its Néron-Severi can not be isometric to $M_{e, 2}^{\prime}$, to $M_{e, 2}$ for an odd $e$ or to $L_{f, 2}$ with $f \in \mathbb{N}_{>0}$. The argument is similar to the one of Proposition 3.10

By Proposition 4.4, if $Y$ is a Nikulin surface, its Néron-Severi group is either isometric to $M_{e, 2}$ or to $M_{2 e, 2}^{\prime}$. By Proposition 4.3 if $X$ is a K3 surface admitting a symplectic involution, its Néron-Severi group is either isometric to $L_{d, 2}$ or to $L_{2 d, 2}^{\prime}$. So if a K3 surface has both properties (i.e. it is in $\mathcal{L}_{2} \cap \mathcal{M}_{2}$ and has Picard 
number 9), its Néron-Severi group is isometric both to a lattice in $\left\{M_{e, 2}, M_{2 e, 2}^{\prime}\right\}$ and to a lattice in $\left\{L_{d, 2}, L_{2 d, 2}^{\prime}\right\}$. Hence we are looking for pairs of lattices, one in $\left\{M_{e, 2}, M_{2 e, 2}^{\prime}\right\}$ and one in $\left\{L_{d, 2}, L_{2 d, 2}^{\prime}\right\}$, which are isometric. If two lattices are isometric, they have the same length. We observe that $l\left(M_{e, 2}\right)=1+l(N)=7$, $l\left(M_{2 e, 2}^{\prime}\right)=1+l(N)-2=5, l\left(L_{d, 2}\right)=1+l\left(\Omega_{2}\right)=9, l\left(L_{2 d, 2}^{\prime}\right)=1+l\left(\Omega_{2}\right)-2=7$. In particular, the unique possible pair of lattices as required is given by $\left(M_{e, 2}, L_{2 d, 2}^{\prime}\right)$. Since if two lattices are isometric they have the same discriminant, one obtains that $e=2 d$.

Corollary 4.7. Two Nikulin surfaces $Y$ and $\hat{Y}$ with Picard number 9 are isogenous by a chain of quotients by involutions if and only if one of the following equivalent conditions hold:

(i) $\mathrm{NS}(Y) \simeq M_{d, 2}, \mathrm{NS}(\hat{Y})=M_{e, 2}$, and there exists $m \in \mathbb{N}_{>0}$ such that either $d=2^{m}$ e or $e=2^{m} d$

(ii) $T_{Y} \simeq U \oplus U \oplus N \oplus\langle-2 d\rangle, T_{\hat{Y}} \simeq U \oplus U \oplus N \oplus\langle-2 e\rangle$ and there exists $m \in \mathbb{N}_{>0}$ such that either $d=2^{m}$ e or $e=2^{m} d$.

Proof. We can assume that $\hat{Y}$ is obtained by iterated quotients from $Y$. Then $Y$ admits a symplectic involution $\sigma$ and, by Theorem 4.6, there exists an even $d$ such that $\operatorname{NS}(Y) \simeq M_{d, 2} \simeq L_{d, 2}^{\prime}$. So $Y$ is the cover of a K3 surface $Z$ with Néron-Severi group $M_{d / 2,2}$ (by Proposition 4.5). If $d$ is odd, then the process stops and $\hat{Y}$ is necessarily $Z$; otherwise, $\mathrm{NS}(Z) \simeq M_{d, 2} \simeq L_{d, 2}^{\prime}$ and $Z$ is the cover of a K3 surface $Z$ with Néron-Severi group $M_{d / 4,2}$. Iterating, if possible, this process $m$ times, one obtains Nikulin surfaces with Néron-Severi group isometric to $M_{d / 2^{m}, 2}$. In particular, one never obtains lattices isometric to $M_{e, 2}^{\prime}$ (for a certain $e$ ) as NéronSeveri groups of a Nikulin surface obtained by iterated quotients from $Y$.

Vice versa, if $\mathrm{NS}(\hat{Y}) \simeq M_{e, 2}$ for a certain $e, \hat{Y}$ is covered by a K3 surface $W$ with $\mathrm{NS}(W) \simeq L_{2 e, e}^{\prime} \simeq M_{2 e, 2}$ (by Proposition 4.5). So $W$ is a Nikulin surface, $2: 1$ covered by a K3 surface with Néron-Severi group isometric to $L_{4 e, e}^{\prime} \simeq M_{4 e, 2}$. Reiterating this process $m$ times one obtains that $\hat{Y}$ is isogenous to a Nikulin surface whose Néron-Severi lattice is isometric to $M_{h, e}$ with $h=2^{m} d$.

The equivalent statement for the transcendental lattice follows by the fact that if the Néron-Severi group of a K3 surface is isometric to $M_{d, 2}$, then its transcendental lattice is isometric to $U \oplus U \oplus N \oplus\langle-2 d\rangle$ (since the discriminant form of the latter is minus the discriminant form of $M_{d, 2}$, and in this case the transcendental lattice is uniquely determined by its genus).

We determined an infinite number of infinite series of Nikulin surfaces of Picard number 9 related by iterated quotients by symplectic involutions. More precisely we proved the following.

Corollary 4.8. For every $d \in \mathbb{N}$, if $\mathrm{NS}(Y) \simeq M_{d, 2}$ there exists an infinite number of $K 3$ surfaces $Y_{m}$ isogenous to $Y$. In particular for each $m$ there exists at least one K3 surface $Y_{m}$ with an isogeny of degree $2^{m}$ to $Y$ whose Néron-Severi group is isometric to $\operatorname{NS}\left(Y_{m}\right)=M_{2^{m} d, 2}$. The transcendental lattice of $Y$ is $T_{Y} \simeq U \oplus U \oplus N \oplus\langle-2 d\rangle$ and for each $m$ the one of $Y_{m}$ is $T_{Y_{m}} \simeq U \oplus U \oplus N \oplus\left\langle-2^{m+1} d\right\rangle$.

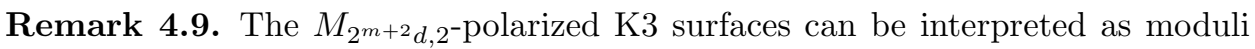

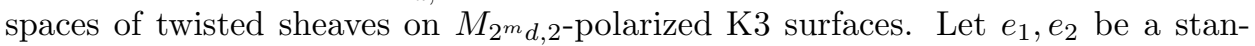
dard basis of the first copy of $U$ inside the K3 lattice $\Lambda_{K 3}$ and choose a primitive 
embedding of $M_{2^{m+2} d, 2}$ in $\Lambda_{K 3}$ so that a generator of $\left\langle 2^{m+1} d\right\rangle$ is $e_{1}+2^{m} d e_{2}$ and $N$ is embedded in $U^{\perp}$. Given $S \in \mathcal{P}\left(M_{2^{m}} d, 2\right)$ generic, the transcendental lattice is $T_{S}=U^{\oplus 2} \oplus N \oplus\left\langle-2^{m+1} d\right\rangle$, and with our previous choice it is easy to see that $\left\langle-2^{m+1} d\right\rangle$ is generated by $t:=e_{1}-2^{m} d e_{2}$. The $B$-field $B=\frac{e_{2}}{2} \in H^{2}(S, \mathbb{Q})$ is a lift for the Brauer class $\beta: T_{S} \rightarrow \mathbb{Z} / 2 \mathbb{Z}$ given by $v \mapsto(v, 2 B)$. It is easy to see that $T(S, B) \cong \operatorname{ker} \beta=U^{\oplus 2} \oplus N \oplus\left\langle-2^{m+3} d\right\rangle \subset H^{*}(S, \mathbb{Z})$, where the last summand is spanned by $(0,2 t, 1)$. Moreover, the orthogonal of $T(S, B)$ inside the Mukai lattice $H^{*}(S, \mathbb{Z})$ is the generalized Picard group $\operatorname{Pic}(S, B)$, which is the sublattice spanned by $f_{1}:=(0,0,1), f_{2}:=\left(2, e_{2}, 0\right), f_{3}:=\left(0, e_{1}+2^{m} d e_{2}, 0\right)$ and $\left(0, b_{i}, 0\right)$ with $b_{1}, \cdots, b_{8} \in M_{2^{m+2} d, 2}$ a basis of the lattice $N$, and has quadratic form

$$
\left(\begin{array}{ccc}
0 & 2 & 0 \\
2 & 0 & 1 \\
0 & 1 & 2^{m+1} d
\end{array}\right) \oplus N .
$$

The isotropic element $v:=2^{m} d f_{2}-f_{3}$ now satisfies $\left((\mathbb{Z} v)^{\perp} \cap \operatorname{Pic}(S, B)\right) / \mathbb{Z} v \simeq$ $M_{2^{m+2} d, 2}$. Hence, the moduli space of stable twisted sheaves $M_{v}(S, \beta)$ is a smooth

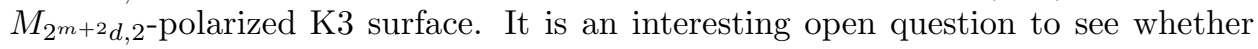
the isogeny of degree 4 which we constructed here coincides with the one induced by a twisted universal family on $S \times M_{v}(S, \beta)$ or not (for further details see Huy, Theorem 0.1]).

4.3. The Galois closure of $2^{2}: 1$ covers. Let $X_{d}$ be a K3 surface such that $\operatorname{NS}\left(X_{d}\right)=M_{d, 2}$. Then $X_{2 d} \in \mathcal{L}_{2} \cap \mathcal{M}_{2}$ and there are the two Galois covers $X_{4 d} \rightarrow-$ $X_{2 d}$ and $X_{2 d} \rightarrow X_{d}$. The composition of these two maps is a $2^{2}: 1$ isogeny, not induced by a Galois cover, by Proposition 3.11, As observed in Remark 3.12 there exist a surface $V$, a group $G \subset \operatorname{Aut}(V)$ and a subgroup $H$ of $G$ such that $V / G$ is birational to $X_{d}$, and $V / H$ is birational to $X_{4 d}$. Here we construct the surface $V$ and the group $G$, proving the following

Proposition 4.10. The group $G$ is the dihedral group of order 8 and $V$ is $a(\mathbb{Z} / 2 \mathbb{Z})^{2}$ Galois cover of $X_{2 d}$, whose branch locus $B$ is the union of 16 smooth rational curves. If $B$ is normal crossing, then $V$ is a positive Kodaira dimension smooth surface such that $h^{1,0}(V)=0$ and $h^{2,0}(V) \geq 35$.

To prove the Proposition one constructs a $(\mathbb{Z} / 2 \mathbb{Z})^{2}$ Galois cover of $X_{2 d}$ (see Section 4.3.1) by a surface denoted by $V$. Then one constructs a $(\mathbb{Z} / 2 \mathbb{Z})^{2}$ Galois cover of $X_{d}$ (see Section 4.3.2), and eventually one proves that these two covers can be pasted to obtain a unique Galois cover by the dihedral group of order 8 (see Section 4.3.3). In order to obtain the $(\mathbb{Z} / 2 \mathbb{Z})^{2}$ covers one compares the branch loci of the $2: 1$ maps $X_{4 d} \rightarrow X_{2 d}$ and $X_{2 d} \rightarrow X_{d}$.

Here we do not consider the Galois closure of $2^{n}: 1$ isogenies given in Corollary 4.8, but for any fixed $n$ a priori one can iterate the previous process.

In the following we will call the $(\mathbb{Z} / 2 \mathbb{Z})^{2}$ Galois covers bidouble covers as in $[\mathrm{C}$, where all the basic definitions and properties of these covers can be found.

4.3.1. A bidouble cover of the surface $X_{2 d}$. Let us denote by $N_{1}, \ldots, N_{8} \subset X_{2 d}$ the rational curves which are the branch locus of the double cover $X_{4 d} \rightarrow-\rightarrow X_{2 d}$. Let us denote by $\sigma_{2 d}$ the symplectic involution on $X_{2 d}$ such that $X_{d}$ is birational to $X_{2 d} / \sigma_{2 d}$. The curves $N_{i}, i=1, \ldots, 8$ are not preserved by $\sigma_{2 d}$, since $\langle H\rangle:=$ $\operatorname{NS}\left(X_{2 d}\right)_{2 d}^{\sigma_{2 d}^{*}}$ is positive definite and more precisely $H^{2}=4 d$. Set $N_{i}^{\prime}:=\sigma_{2 d}\left(N_{i}\right)$. 
Hence we found two even sets of eight rational curves on $X_{2 d}:\left\{N_{1}, \ldots, N_{8}\right\}$ and $\left\{N_{1}^{\prime}, \ldots, N_{8}^{\prime}\right\}$. Let $D_{1}:=\sum_{i=1}^{8} N_{i}, D_{2}:=\sum_{i=1}^{8} N_{i}^{\prime}, D_{3}:=0$ and $2 L_{i}:=D_{j}+$ $D_{k}, i, j, k \in\{1,2,3\}$. The six divisors $D_{j}, L_{i}, j, i=1,2,3$ in $\operatorname{NS}\left(X_{2 d}\right)$ satisfy the conditions which define a bidouble cover, so there exists a surface $V$ such that $(\mathbb{Z} / 2 \mathbb{Z})^{2} \in \operatorname{Aut}(V)$ and $V /(\mathbb{Z} / 2 \mathbb{Z})^{2}$ is (birational to) $X_{2 d}$ (see $[\mathrm{C}$, Section $2]$ ). Moreover, there are three surfaces which are double covers of $X_{2 d}$ branched respectively along the curves supported on $2 L_{1}, 2 L_{2}$ and $2 L_{3}$; all of them are $2: 1$ covered by $V$. Since $L_{2}=\sum_{i=1}^{8} N_{i} / 2$, the double cover of $X_{2 d}$ branched on $2 L_{2}$ is a non-minimal model of $X_{4 d}$. We denote the cover branched on the curves in the support of $2 L_{2}$ by $\widetilde{X_{4 d}}$. Similarly the double cover of $X_{2 d}$ branched on $\cup_{i} N_{i}^{\prime}$ is the blow up of a K3 surface, $X_{4 d}^{\prime}$, in 8 points and it will be denoted by $\widetilde{X_{4 d}^{\prime}}$. The Néron-Severi group of the K3 surface $X_{4 d}^{\prime}$ is determined by the one of $X_{2 d}$, by Proposition 4.5, and thus it is isometric to $M_{4 d}$. We obtain the following diagram:

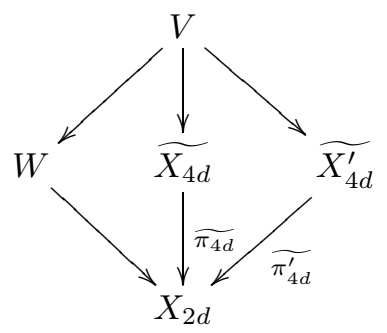

The surfaces $W$ and $V$ have non negative Kodaira dimension, because they are covers of K3 surfaces.

Let us now suppose that the intersections $N_{i} \cap N_{j}^{\prime}$ are transversal, and thus both the branch divisors of $W \rightarrow X_{2 d}$ and of $V \rightarrow X_{2 d}$ are normal crossing. Under this assumption, $V$ is smooth and the birational invariants of $W$ and $V$ depend only on $L_{j}^{2}$, for $j=1,2,3$. The surface $W$ is the double cover of $X_{2 d}$ branched on the reducible curve which is the support of $2 L_{3}$, i.e. on the curve $\bigcup_{i=1}^{8}\left(N_{i} \cup N_{i}^{\prime}\right)$. Since $N_{i}+N_{i}^{\prime}$ is an effective $\left(\sigma_{2 d}^{*}\right)$-invariant divisor and $H$ is the ample generator of $\operatorname{NS}\left(X_{2 d}\right)^{\sigma_{2 d}^{*}}$, there exists a positive integer $k_{i}$ such that $N_{i}+N_{i}^{\prime}=k_{i} H$. Then $L_{3}^{2}=\left(\sum_{i=1}^{8} k_{i} H\right)^{2} / 4=d\left(\sum_{i=1}^{8} k_{i}\right)^{2}$ and

$$
\chi(W)=4+\frac{d}{2}\left(\sum_{i=1}^{8} k_{i}\right)^{2}, h^{2,0}(W)=3+\frac{d}{2}\left(\sum_{i=1}^{8} k_{i}\right)^{2} \text { and } h^{1,0}(W)=0 .
$$

The singularities of $W$ are in the inverse image of the singular points of $\bigcup_{i=1}^{8}\left(N_{i} \cup\right.$ $\left.N_{i}^{\prime}\right)$ and $V$ is a double cover of $W$ branched on its singular points. The invariants of $V$ can be computed by [C, Section 2], from which one obtains

$$
h^{2,0}(V)=h^{2,0}(W)=3+\frac{d}{2}\left(\sum_{i=1}^{8} k_{i}\right)^{2} \geq 3+32 d \geq 35, \quad h^{1,0}(V)=h^{1,0}(W)=0 .
$$

Hence $V$ is a surface with non negative Kodaira dimension, $h^{2,0}(V) \geq 35$ and $h^{1,0}(V)=0$, so its Kodaira dimension is necessarily positive. 
4.3.2. A bidouble cover of the surface $X_{d}$. The surface $X_{d}$ is the desingularization of the quotient of $X_{2 d} / \sigma_{2 d}$ and we will denote by $R_{1}, \ldots, R_{8}$ the eight disjoint rational curves resolving the singularities of $X_{2 d} / \sigma_{2 d}$. Equivalently, the double cover of $X_{d}$ branched on $\bigcup_{i} R_{i}$ is birational to $X_{2 d}$. Denoted by $\pi_{2 d}: X_{2 d} \rightarrow X_{2 d} / \sigma_{2 d}$ the quotient map, one observes that $\pi_{2 d}\left(N_{i}\right)=\pi_{2 d}\left(N_{i}^{\prime}\right), i=1, \ldots, 8$, and $\pi_{2 d}\left(N_{i}\right)$ is a rational curve singular in the points $\pi_{2 d}\left(N_{i} \cap N_{i}^{\prime}\right)$. We denote by $\overline{N_{i}}$ the strict transform on $X_{d}$ of the curve $\pi_{2 d}\left(N_{i}\right)$. The curves $\overline{N_{i}}$ could be singular and the set $\left\{\overline{N_{1}}, \ldots, \overline{N_{8}}\right\}$ is a divisible set. Moreover, since $N_{i}+N_{i}^{\prime}=k_{i} H \subset \mathrm{NS}\left(X_{2 d}\right)^{\sigma_{2 d}^{*}}$, one has $\left(\pi_{2 d}\right)_{*}\left(N_{i}+N_{i}^{\prime}\right) \subset \operatorname{NS}\left(X_{2 d} / \sigma_{2 d}\right)$. Hence $\overline{N_{i}} \subset\left(N^{\left.\perp_{\mathrm{NS}\left(X_{d}\right)}\right)}\right)$.

The sets $\left\{R_{1}, \ldots, R_{8}\right\}$ and $\left\{\overline{N_{1}}, \ldots \overline{N_{8}}\right\}$ are two 2-divisible sets of curves, which allow us to construct a bidouble cover of $X_{d}$, whose data are $\Delta_{1}:=\sum_{i=1}^{8} R_{i}$, $\Delta_{2}:=\sum_{i=1}^{8} \overline{N_{i}}, \Delta_{3}:=\sum_{i=1}^{8}\left(R_{i}+\bar{N}_{i}\right), 2 \Gamma_{i}:=\Delta_{j}+\Delta_{k}$, with $\{i, j, k\}=\{1,2,3\}$. The double cover $\widetilde{X_{2 d}} \rightarrow X_{d}$ is branched over $\cup_{i} R_{i}$, i.e. the curve in the support of $2 \Gamma_{2}$. It induces a double cover of $\widetilde{X_{2 d}}$ branched over $\cup_{i}\left(\widetilde{N_{i}}+\widetilde{N_{i}^{\prime}}\right)$, where $\widetilde{N_{i}}$ (resp. $\widetilde{N_{i}^{\prime}}$ ) is the strict transform on $\widetilde{X_{2 d}}$ of the curve $N_{i}$ (resp. $N_{i}^{\prime}$ ). Let us denote by $\widetilde{W}$ the surface double cover of $\widetilde{X_{2 d}}$ branched on $\cup_{i}\left(\widetilde{N_{i}}+\widetilde{N_{i}^{\prime}}\right)$. So we have the following diagram:

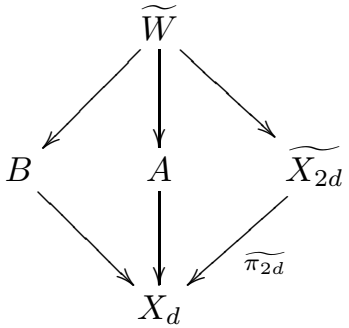

where $\widetilde{\pi_{2 d}}: \widetilde{X_{2 d}} \rightarrow X_{d}$ is induced by $\pi_{2 d}$.

4.3.3. The $\mathcal{D}_{4}$ cover of $X_{d}$. Both the diagrams (4.2) and (4.1) induce a $2: 1$ rational map $W \rightarrow X_{2 d}$, which is (birationally) the double of cover of $X_{2 d}$ branched on $\bigcup_{i=1}^{8}\left(N_{i} \cup N_{i}^{\prime}\right)$. Hence these diagrams can be pasted to obtain the following, where all the arrows are rational maps of generically degree 2

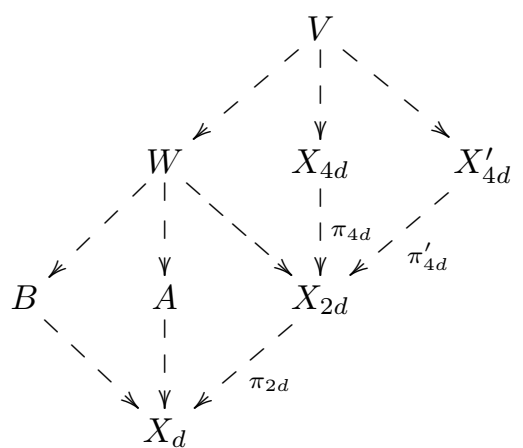

We already proved that the $4: 1$ covers $X_{4 d} \rightarrow X_{d}$ and $X_{4 d}^{\prime} \rightarrow X_{d}$ are not Galois covers in Proposition 3.11. On the other hand, the cover $4: 1 W \rightarrow X_{d}$ is a Galois 
cover (indeed a bidouble cover), by construction. Since $V \rightarrow X_{2 d}$ is constructed as bidouble cover, the cover involution of the cover $W \rightarrow X_{2 d}$, lifts to an involution of $V$ (which is the cover involution of $V \rightarrow X_{4 d}$ ). Hence one obtains that the cover $V \rightarrow X_{d}$ is a Galois $8: 1$ cover. The cover group $G$ is an order 8 group, which admits non normal subgroups of order 2 (otherwise $X_{4 d} \rightarrow X_{d}$ should be a Galois cover). Hence $G \simeq \mathcal{D}_{4}$, the dihedral group of order 8. We recall that $\mathcal{D}_{4}:=<s, r \mid s^{2}=1, r^{4}=1, r s=s r^{-1}>$. The center $H$ of $G$ is $H:=<r^{2}>$ and the quotient of $V$ by $H$ is birational to $W$. So we conclude that the Galois cover is given by the surface $V$ on which acts the group $G=\mathcal{D}_{4}$.

4.4. The K3 surface $X_{2} \in \mathcal{L}_{2} \cap \mathcal{M}_{2}$. By Proposition 4.6, for every even $d$, if a K3 surface $X_{d}$ is such that $\mathrm{NS}\left(X_{d}\right) \simeq L_{d, 2}^{\prime}$, then $X_{d}$ admits a symplectic involution and it is $2: 1$ cyclically covered by a K3 surface. Here we describe geometrically these properties for the minimum possible value of $d$, i.e. for $d=2$ : let $X_{2}$ be a K3 surface with $\mathrm{NS}\left(X_{2}\right) \simeq L_{2,2}^{\prime}$. It admits an involution $\sigma$ and by Proposition 4.5 the K3 surface $Y_{1}$ which is the desingularization of $X_{2} / \sigma$ is such that $\mathrm{NS}\left(Y_{1}\right)=M_{1,2} \simeq\langle 2\rangle \oplus N$. Since $\mathrm{NS}\left(X_{2}\right) \simeq M_{2,2} \simeq\langle 4\rangle \oplus N$ (by Proposition 4.6), the surface $X_{2}$ is $2: 1$ covered by a K3 surface $X_{4}$, whose Néron-Severi group is $\mathrm{NS}\left(X_{4}\right) \simeq L_{4,2}^{\prime}$ (by Proposition 4.5). Since $X_{2}$ is a Nikulin surface, there are 8 disjoint rational curves, which resolve the singularities of the quotient of $X_{4}$ by a symplectic involution. Thus the surface $X_{2}$ admits two different descriptions according to the interpretation of it as K3 surface with a symplectic involution or as Nikulin surface. These descriptions are associated to different projective models, induced by different (pseudo)ample divisors. Here we recall these descriptions and we explain how to pass from one to the other.

By [vGS, Section 3.5], any K3 surface $X_{2}$ such that $\operatorname{NS}\left(X_{2}\right) \simeq L_{2,2}^{\prime}$ is described as bidouble cover of $\mathbb{P}^{2}$ as follows: one considers two smooth plane curves $B$ and $C_{0}$ of degree respectively 4 and 2 in $\mathbb{P}^{2}$. The double cover of $\mathbb{P}^{2}$ branched on $B \cup C_{0}$ is a surface singular in eight points, the inverse image of $B \cap C_{0}$. The resolution of this surface is the $\mathrm{K} 3$ surface $X_{1}$ such that $\mathrm{NS}\left(X_{1}\right) \simeq M_{1,2}$ and the eight rational curves arising from this resolution will be denoted by $R_{i}, i=1, \ldots, 8$. The curves $R_{1}, \ldots, R_{8}$ form an even set of rational curves on $X_{1}$ and the double cover of $X_{1}$ branched on $\cup_{i} R_{i}$ is, by construction, a K3 surface $X_{2}$ such that $\mathrm{NS}\left(X_{2}\right) \simeq L_{2,2}^{\prime}$. The choice of the curves $B \cup C_{0}$ totally determines the surfaces $X_{1}$ and $X_{2}$. To construct the bidouble cover one considers also the double cover of $\mathbb{P}^{2}$ branched on $C_{0}$ and the double cover of $\mathbb{P}^{2}$ branched on $B$. The first surface is a quadric $Q \simeq \mathbb{P}^{1} \times \mathbb{P}^{1} \subset \mathbb{P}^{3}$, the latter a del Pezzo surface of degree 2 , denoted in the following by $d P$. Hence one has the following diagram, where all the arrows are rational maps of degree 2 :

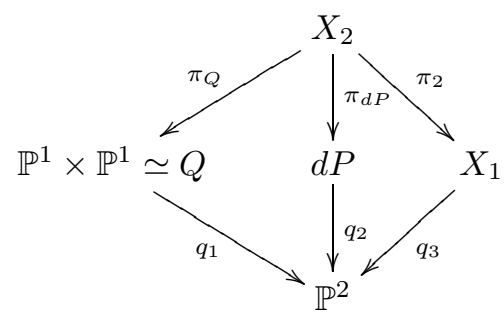


The Néron-Severi group of $X_{2}$ is isometric to $L_{2,2}^{\prime}$, hence it is an overlattice of index two of $\langle 4\rangle \oplus E_{8}(-2)$. The linear system of the ample divisor $L$, orthogonal to $E_{8}(-2)$ in $L_{2,2}^{\prime}$ exhibits $X_{2}$ as double cover of a quadric $\mathbb{P}^{1} \times \mathbb{P}^{1}$ in $\mathbb{P}^{3}$. One can assume that the class generating $L_{2,2}^{\prime} / L_{2,2}$ is $E_{1}:=\left(L+e_{1}\right) / 2$, where $e_{i}$ is a standard basis of $E_{8}(-2)$ (i.e. $e_{i} e_{i+1}=2$ if $i=1, \ldots 6, e_{3} e_{8}=2,\left(e_{i}\right)^{2}=-4$ and the other intersections are 0$)$. Then the divisor $E_{1}$ is a nef divisor and the map associated to its linear system $\varphi_{\left|E_{1}\right|}: X_{2} \rightarrow \mathbb{P}^{1}$ is a genus 1 fibration. The action of $\sigma^{*}$ on $\mathrm{NS}\left(X_{2}\right)$ is the identity on the subspace $\langle L\rangle$ and minus the identity on the subspace $L^{\perp} \simeq E_{8}(-2)$. So the image of $E_{1}$ by $\sigma^{*}$ is the nef divisor $E_{2}:=\left(L-e_{1}\right) / 2=E_{1}-e_{1}$ (see [VGS, Section 3.5]). The two maps $\varphi_{\left|E_{i}\right|}, i=1,2$ are the maps on the rulings of the quadric $Q \subset \mathbb{P}^{3}$ image of the map $\varphi_{|L|}=\varphi_{\left|E_{1}+E_{2}\right|}$. In particular the set of divisors $\left\{E_{1}, e_{1}, \ldots e_{8}\right\}$ is a basis of $\operatorname{NS}\left(X_{2}\right)$.

By (4.4), it follows that $X_{2}$ admits three commuting involutions, the covering involutions of the three double covers $\pi_{Q}, \pi_{d P}, \pi_{2}$. The latter involution is the symplectic involution $\sigma$, the others will be denoted by $\iota_{Q}$ and $\iota_{d P}$ respectively.

Proposition 4.11. The involutions $\iota_{Q}$ and $\iota_{d P}$ are non-symplectic involutions and their composition is the symplectic involution $\sigma$. The group $\left\langle\iota_{Q}, \iota_{d P}\right\rangle$ is isomorphic to $(\mathbb{Z} / 2 \mathbb{Z})^{2}$ and it is the Galois group of the $2^{2}: 1$ cover $\pi: X_{2} \rightarrow \mathbb{P}^{2}$.

The induced three involutions on $\mathrm{NS}\left(X_{2}\right)$ act as follows on the basis $\left\{E_{1}, e_{1}, \ldots e_{8}\right\}$ :

$$
\begin{array}{llll}
\sigma^{*}\left(E_{1}\right)=E_{1}-e_{1}, & \sigma^{*}\left(e_{i}\right)=-e_{i}, & i=1, \ldots, 8 \\
\iota_{Q}^{*}\left(E_{1}\right)=E_{1}, & \iota_{Q}^{*}\left(e_{1}\right)=e_{1}, & \iota_{Q}^{*}\left(e_{2}\right)=-e_{1}-e_{2}, & \iota_{Q}^{*}\left(e_{j}\right)=-e_{j}, \\
\iota_{d P}^{*}\left(E_{1}\right)=E_{1}-e_{1}, & \iota_{d P}^{*}\left(e_{1}\right)=-e_{1}, & \iota_{d P}^{*}\left(e_{2}\right)=e_{1}+e_{2} & \iota_{d P}^{*}\left(e_{j}\right)=e_{j},
\end{array}
$$

where $j=3, \ldots, 8$.

Proof. The action of $\sigma$ is minus the identity on $\left(\mathrm{NS}\left(X_{2}\right)^{\sigma}\right)^{\perp} \simeq E_{8}(-2) \subset \mathrm{NS}\left(X_{2}\right)$ and we chose the basis of $\mathrm{NS}\left(X_{2}\right)$ in such a way that the divisors $e_{i}, i=1, \ldots, 8$ span exactly $\left(\operatorname{NS}\left(X_{2}\right)^{\sigma}\right)^{\perp} \simeq E_{8}(-2)$. Moreover we chose $L$ to be the orthogonal to $\left\langle e_{i}\right\rangle_{i=1, \ldots 8}$ and thus $\sigma^{*}(L)=L$. By the definition of $E_{1}\left(=\left(L+e_{1}\right) / 2\right)$ one obtains $\sigma^{*}\left(E_{1}\right)=\left(L-e_{1}\right) / 2=E_{1}-e_{1}$.

The automorphism $\iota_{Q}$ is such that $X_{2} / \iota_{Q}$ is a rational surface and thus $\iota_{Q}$ is non symplectic and $X_{2} / \iota_{Q}$ is smooth. Since $X_{2} / \iota_{Q}$ is $\mathbb{P}^{1} \times \mathbb{P}^{1}, \operatorname{rank}\left(\operatorname{NS}\left(X_{2}\right)^{\iota_{Q}}\right)=2$ and $\mathrm{NS}\left(X_{2}\right)^{\iota Q}$ is generated by the divisors which induce the maps $X_{2} \rightarrow \mathbb{P}^{1}$ given by the composition of the quotient map $\pi_{Q}: X_{2} \rightarrow \mathbb{P}^{1} \times \mathbb{P}^{1}$ with the projection on the first, respectively second, factor. These maps are $\varphi_{\left|E_{1}\right|}: X_{2} \rightarrow \mathbb{P}^{1}$ and $\varphi_{\left|E_{2}\right|}: X_{2} \rightarrow \mathbb{P}^{1}$. So $\operatorname{NS}\left(X_{2}\right)^{\iota_{Q}}=\left\langle E_{1}, E_{2}\right\rangle$ and $\iota_{Q}$ acts as minus the identity on $\left(\operatorname{NS}\left(X_{2}\right)^{\iota}\right)^{\perp}$. So $\iota_{Q}^{*}\left(e_{j}\right)=-e_{j}$ if $j=3, \ldots, 8, \iota_{Q}^{*}\left(E_{1}\right)=E_{1}$, and $\iota_{Q}^{*}\left(E_{2}\right)=\iota_{Q}^{*}\left(E_{1}-\right.$ $\left.e_{1}\right)=E_{1}-\iota_{Q}^{*}\left(e_{1}\right)=E_{1}-e_{1}=E_{2}$. It follows $\iota_{Q}^{*}\left(e_{1}\right)=e_{1}$. In order to find the image of $e_{2}$ it suffices to recall that $\iota_{Q}^{*}$ is an involution and that $\left(\iota_{Q}^{*}\left(e_{2}\right)\right) \cdot\left(\iota_{Q}^{*}(D)\right)=e_{2} D$ for any divisor $D \in \operatorname{NS}\left(X_{2}\right)$. The group $\left\langle\iota_{Q}, \sigma\right\rangle$ is by construction the Galois group of the cover $\pi: X_{2} \rightarrow \mathbb{P}^{2}$, so it is isomorphic to $(\mathbb{Z} / 2 \mathbb{Z})^{2}$ and contains three different involutions, each of them is the composition of the other two. In particular $\iota_{d P}=\iota_{Q} \circ \sigma$ and so $\iota_{d P}^{*}=\sigma^{*} \circ \iota_{Q}^{*}$ and $\iota_{d P}$ is non-symplectic.

We already observed that the classes $E_{1}:=\left(L+e_{1}\right) / 2$ and $E_{2}:=\left(L-e_{1}\right) / 2$ induce two elliptic fibrations $\varphi_{\left|E_{i}\right|}: X_{2} \rightarrow \mathbb{P}^{1}$. By the properties of these elliptic fibrations we will be able to identify the classes of irreducible rational curves on $X_{2}$ and in particular 8 classes which span the Nikulin lattice. The following proposition gives 
the explicit isometry between $L_{2,2}^{\prime}$ and $M_{2,2}$ and shows directly that the surface $X_{2}$ admits a 2:1 rational double cover by another K3 surface, thus it provides an explicit geometric interpretation of Theorem 3.9 in the case $n=2$.

Proposition 4.12. Both the genus 1 fibrations $\varphi_{\left|E_{1}\right|}: X_{2} \rightarrow \mathbb{P}^{1}$ and $\varphi_{\left|E_{2}\right|}: X_{2} \rightarrow$ $\mathbb{P}^{1}$ have no reducible fibers and 8 disjoint sections which can be chosen to generate the Mordell-Weil group (which is isomorphic to $\mathbb{Z}^{7}$ ). One can choose these sections, for each fibration, in such a way that 7 sections are in common, the eighth section of $\varphi_{\left|E_{1}\right|}$ is a 5-section for $\varphi_{\left|E_{2}\right|}$ and vice versa the eighth section of $\varphi_{\left|E_{2}\right|}$ is a 5-section for $\varphi_{\left|E_{1}\right|}$. The eight sections of $\varphi_{\left|E_{1}\right|}$ (resp. $\varphi_{\left|E_{2}\right|}$ ) chosen as above form an even set of eight disjoint rational curves, so $X_{2}$ is a Nikulin surface and $\mathrm{NS}\left(X_{2}\right) \simeq M_{2,2}$.

Proof. Since one has a basis of $\mathrm{NS}\left(X_{2}\right)$, one can compute explicitly the sublattice $E_{1}^{\perp}:=\left\{D \in \mathrm{NS}\left(X_{2}\right) \simeq L_{2,2}^{\prime} \mid D E_{1}=0\right\}$ and one observes that it is $P(2)$ for a certain degenerate even lattice $P$. In particular there are no $(-2)$-classes orthogonal to $E_{1}$ in $\mathrm{NS}\left(X_{2}\right)$ and thus the fibration $\varphi_{\left|E_{1}\right|}$ does not admit reducible fibers. The fibration $\varphi_{\left|E_{2}\right|}: X_{2} \rightarrow \mathbb{P}^{1}$ is the image of $\varphi_{\left|E_{1}\right|}$ for the automorphism $\sigma$, so also $\varphi_{\left|E_{2}\right|}$ has no reducible fibers too.

To conclude the proof it suffices to exhibit the classes of the irreducible rational curves with the required properties. Let us assume that $N_{i}$ is a class such that $N_{i}^{2}=-2, N_{i} L>0, N_{i} E_{1}=1$. Then $N_{i}$ is the class of an effective divisor (by $N_{i} L>0$ ), supported on a (possibly reducible) curve. If $N_{i}$ is irreducible, then it is a section of $\varphi_{\left|E_{1}\right|}$. Otherwise it should be the sum of a section and some irreducible components of reducible fibers, but there are no reducible fibers in the genus 1 fibration $\varphi_{\left|E_{1}\right|}$. So $N_{i}$ is a section of $\varphi_{\left|E_{1}\right|}$. All the classes listed below satisfy the conditions $N_{i}^{2}=-2, N_{i} L>0, N_{i} E_{1}=1$, so they are supported on irreducible rational curves, all sections of $E_{1}$ :

$$
\begin{aligned}
& N_{1}=E_{1}+e_{2} ; \quad N_{2}=E_{1}+e_{2}+e_{3} ; \\
& N_{3}=E_{1}+e_{2}+e_{3}+e_{4} ; \quad N_{4}=E_{1}+e_{2}+e_{3}+e_{4}+e_{5} ; \\
& N_{5}=E_{1}+e_{2}+e_{3}+e_{4}+e_{5}+e_{6} ; \quad N_{6}=E_{1}+e_{2}+e_{3}+e_{4}+e_{5}+e_{6}+e_{7} ; \\
& N_{7}=E_{1}-2 e_{1}-3 e_{2}-5 e_{3}-4 e_{4}-3 e_{5}-2 e_{6}-e_{7}-3 e_{8} ; \quad N_{8}=3 E_{1}+e_{2}-e_{8} .
\end{aligned}
$$

Since $N_{i} N_{j}=0$ for every $i, j=1, \ldots, 8, i \neq j$, the curves $N_{i}$ are disjoint. Moreover $\left(\sum_{i=1}^{8} N_{i}\right) / 2 \in \mathrm{NS}\left(X_{2}\right)$, so $\left\{N_{1}, \ldots, N_{8}\right\}$ is an even set of disjoint rational curves and thus there is a $2: 1$ cover branched on these rational curves, i.e. $X_{2} \in \mathcal{M}_{2}$.

The curves $N_{i} i=1, \ldots 7$ intersect both $E_{1}$ and $E_{2}$ in 1 point. So the fibrations $\varphi_{\left|E_{1}\right|}$ and $\varphi_{\left|E_{2}\right|}$ share 7 sections.

The divisor $H:=6 E_{1}-e_{1}+2 e_{2}-2 e_{8}$ is a pseudoample divisor of self intersection 4 which is orthogonal to all the $N_{i}$ 's. So $N\left(X_{2}\right) \simeq M_{2,2}$.

The class $N_{8}^{\prime \prime}:=3 E_{1}-2 e_{1}+e_{2}-e_{8}$ is a section of $\varphi_{\left|E_{2}\right|}$ and a 5 section of $\varphi_{\left|E_{1}\right|}$. The class $\left(\sum_{i=1}^{7} N_{i}+N_{8}^{\prime \prime}\right) / 2 \in \mathrm{NS}\left(X_{2}\right)$, so $\left\{N_{1}, N_{2}, N_{3}, N_{4}, N_{5}, N_{6}, N_{7}, N_{8}^{\prime \prime}\right\}$ is an even set of disjoint rational curves.

The explicit knowledge of the change of bases from $\left\{E_{1}, e_{1}, \ldots e_{8}\right\}$ to $\left\{H, N_{1}, \ldots, N_{7}, \sum_{i=1}^{8} N_{i} / 2\right\}$ given in the proof of Proposition 4.12 allows one to obtain some interesting geometric characterizations of the K3 surfaces with $\mathrm{NS}\left(X_{2}\right) \simeq L_{2,2}^{\prime}$. Indeed, let $S$ be a K3 surface of Picard number 9 and which satisfies one of the following conditions:

- $S$ admits an elliptic fibration $\mathcal{E}: S \rightarrow \mathbb{P}^{1}$ without reducible fibers and admitting 8 disjoint sections, $P_{1}, \ldots, P_{8}$, such that $\left(\sum_{i} P_{i}\right) / 2 \in \mathrm{NS}(S)$. 
- $S$ admits an elliptic fibration $\mathcal{E}: S \rightarrow \mathbb{P}^{1}$ without reducible fibers with zero section $O$. The Mordell-Weil group of $\mathcal{E}$ is generated by 7 sections, $P_{1}, \ldots, P_{7}$, such that $\left\{O, P_{1}, \ldots, P_{6}\right\}$ are mutually disjoint and $P_{7}$ intersects the zero section in 12 points and the other sections $P_{i}, i=1, \ldots, 6$ in 6 points.

- $S$ admits two elliptic fibrations $\mathcal{E}$ and $\mathcal{F}$ with class of the fiber $E$ and $F$ respectively such that $E F=2$. Let us assume that there are 7 orthogonal rational curves such that 6 are sections of both the fibrations, the seventh is section of one fibration and a 5 -section for the others.

Then $S$ satisfies also the other conditions, it admits a symplectic involution switching $\mathcal{E}$ and $\mathcal{F}$ and $S$ is a Nikulin surface. In particular $\operatorname{NS}\left(X_{2}\right) \simeq L_{2,2}^{\prime} \simeq M_{2,2}$. Indeed, any of the above set of data of fibrations and sections is enough to exhibit the lattice $L_{2,2}^{\prime}$ as the Néron-Severi group of $X_{2}$, as it follows by the proof of Proposition 4.12 .

The map $\varphi_{|H|}: X_{2} \rightarrow \mathbb{P}^{3}$ exhibits $X_{2}$ as a singular quartic in $\mathbb{P}^{3}$ and its eight nodes are $\varphi_{|H|}\left(N_{i}\right)$ for $i=1, \ldots, 8$. It is well known that the projection of a nodal quartic from a node gives a model of the same K3 surface as a double cover of $\mathbb{P}^{2}$ branched on a sextic. In particular, let us consider the projection by the node $\varphi_{|H|}\left(N_{8}\right)$, induced by the linear system $\left|H-N_{8}\right|$. We thus have a $2: 1$ map $\varphi_{\left|H-N_{8}\right|}: X_{2} \rightarrow \mathbb{P}^{2}$, which contracts the 7 curves $N_{i}$ to seven nodes of the branch sextic. Hence we obtain the following diagram, where the vertical arrows are contractions of 7 curves and the horizontal arrows are $2: 1$ maps:

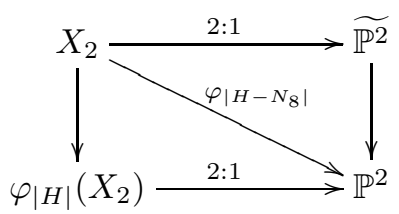

In particular $\widetilde{\mathbb{P}^{2}}$ is the blow up of $\mathbb{P}^{2}$ in the seven points $\varphi_{\left|H-N_{8}\right|}\left(N_{i}\right)$ (which are the singular points of the branch locus of the map $X_{2} \rightarrow \mathbb{P}^{2}$ ) and thus $\widetilde{\mathbb{P}^{2}}$ is a del Pezzo surface of degree 2. The cover involution of the double cover $X_{2} \rightarrow \widetilde{\mathbb{P}}^{2}$ is an involution $i$, such that $i^{*}\left(H-N_{8}\right)=H-N_{8}, i^{*}\left(N_{i}\right)=N_{i}, i=1, \ldots 7$ and $\iota^{*}\left(N_{8}\right)=2 H-3 N_{8}$. One is now able to rewrite the action of $i^{*}$ on the basis $\left\{L, e_{1} \ldots, e_{8}\right\}$ and one finds that $i^{*}=\iota_{d P}^{*}$ (where $\iota_{d P}^{*}$ is as in Proposition 4.11). Thus, we the notation of (4.4), one obtains $\widetilde{\mathbb{P}^{2}}=d P, \iota_{d P}=i$ and the map $\pi_{d P}$ is induced by the projection of $\varphi_{|H|}\left(X_{2}\right)$ from the node $\varphi_{|H|}\left(N_{8}\right)$. The even set $\left\{N_{1}, \ldots, N_{7}, N_{8}^{\prime \prime}\right\}$ is nothing but the image of the even set $\left\{N_{1}, \ldots, N_{8}\right\}$ for the action of $\iota_{d P}$.

In Section 4.3 we proved that the construction of the $\mathcal{D}_{4}$ Galois cover of $X_{d}$ is totally determined by two sets of rational curves in $X_{d}$, i.e. the sets $\left\{R_{1}, \ldots, R_{8}\right\}$ and $\left\{\bar{N}_{1}, \ldots, \bar{N}_{8}\right\}$. In particular in the case we are now considering, i.e. if $d=1$, the curves $R_{i}, i=1, \ldots, 8$ were already considered in the diagram (4.4) and are mapped by $q_{3}: X_{1} \rightarrow \mathbb{P}^{2}$ to the eight singular points of the branch sextic $B \cap C_{0}$. We now describe the curves $\bar{N}_{i}$, by giving their image as plane curves $q_{3}\left(\overline{N_{i}}\right) \subset \mathbb{P}^{2}$. 
By construction, $q_{3}\left(\overline{N_{i}}\right)=\pi\left(N_{i}\right)$, where $\pi: X_{2} \rightarrow \mathbb{P}^{2}$ is the rational $2^{2}: 1$ map given in (4.4).

Proposition 4.13. For each $i=1, \ldots, 7$, the curve $\pi\left(N_{i}\right) \subset \mathbb{P}^{2}$ is a bitangent line to the quartic $B$. The curve $\pi\left(N_{8}\right)$ is a rational irreducible sextic $D \subset \mathbb{P}^{2}$ which is tangent to $B \cup C_{0}$ in all their intersection points. The curves $\pi\left(N_{i}\right), i=1, \ldots, 8$ split in $X_{1}$, the orbit of $N_{i}$ with respect to $\left\langle\iota_{Q}, \iota_{d P}\right\rangle$ consists of two rational curves if $i=1, \ldots, 7$ and of the four curves if $i=8$.

Proof. The surface $d P$ is a degree 2 del Pezzo surface, and then it is naturally endowed with an involution $i$, which is the cover involution of the $2: 1$ map $d P \rightarrow \mathbb{P}^{2}$ (see [DO, Chaptes VII, Section 4] for details on del Pezzo surfaces of degree 2 and its involution). The double cover $q_{2}: d P \rightarrow \mathbb{P}^{2}$ is branched on $B \subset \mathbb{P}^{2}$. Since $d P$ is a del Pezzo surface of degree 2, there is a set of 7 disjoint $(-1)$-curves on $d P$, denoted by $p_{i}, i=1, \ldots, 7$ and such that $\beta_{d P}: d P \rightarrow \mathbb{P}^{2}$ is a contraction of these $(-1)$-curves. The plane curves $q_{2}\left(p_{i}\right) \subset \mathbb{P}^{2}$ are 7 lines which are bitangent to $B$, and each of them splits in the double cover into two rational curves $p_{i}, i\left(p_{i}\right)$, $i=1, \ldots, 7$. So we have the following commutative diagram:

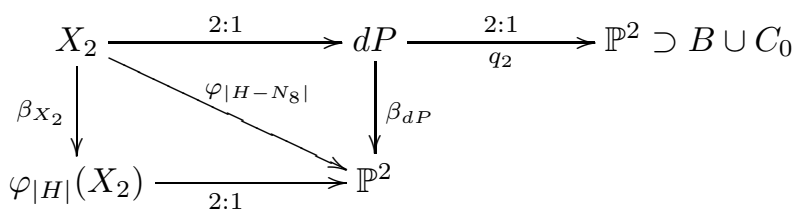

where $\beta_{X_{2}}$ contracts the curves $N_{i}, i=1, \ldots, 8$. For $i=1, \ldots, 7$, one has $\varphi_{\left|H-N_{8}\right|}\left(N_{i}\right)=$ $\beta_{d P}\left(p_{i}\right)$. Each of the 7 lines $q_{2}\left(p_{i}\right) \subset \mathbb{P}^{2}$ is bitangent to $B$, and intersects $C_{0}$ transversally. So $q_{2}\left(p_{i}\right)$ does not split in the double cover $q_{1}: Q \rightarrow \mathbb{P}^{2}$, which is branched on $C_{0}$. In particular, $q_{1}^{-1}\left(q_{2}\left(p_{i}\right)\right)=q_{1}^{-1}\left(\pi\left(N_{i}\right)\right)$ is an irreducible smooth rational curve for $i=1, \ldots, 7$. So, for each $i=1, \ldots, 7, \pi^{-1}\left(\pi_{d P}\left(p_{i}\right)\right) \subset X_{2}$ consists of a pair of rational curves, switched by $\iota_{Q}$, preserved by $\iota_{d P}$ and then switched by $\sigma=\iota_{Q} \circ \iota_{d P}$. This can also be checked directly on the classes of the curves $N_{i}$ by using Propositions 4.11 and 4.12, indeed $\iota_{Q}^{*}\left(N_{i}\right)=N_{i}$ and $\iota_{d P}\left(N_{i}\right)=\sigma\left(N_{i}\right) \neq N_{i}$ for $i=1, \ldots, 7$. Since for each $i \in\{1, \ldots, 7\}$ the curve $N_{i}$ is a section of both the elliptic fibrations $\left|E_{1}\right|$ and $\left|E_{2}\right|$ on $X_{2}$, the curve $q_{1}\left(N_{i}\right) \subset Q \simeq \mathbb{P}^{1} \times \mathbb{P}^{1}$ is a curve of bidegree $(1,1)$ if $i=1, \ldots 7$.

It remains to describe the curve $N_{8} \subset X_{2}$. The orbit of $N_{8}$ is given by the four classes $N_{8}=3 E_{1}+e_{2}-e_{8}, N_{8}^{\prime}:=\sigma\left(N_{8}\right)=3 E_{1}-3 e_{1}-e_{2}+e_{8} ; N_{8}^{\prime \prime}=$ $\iota_{d P}\left(N_{8}\right)=3 E_{1}-2 e_{1}+e_{2}-e_{8}, N_{8}^{\prime \prime \prime}:=\sigma\left(\iota_{d P}\left(N_{8}\right)\right)=3 E_{1}-e_{1}-e_{2}+e_{8}$. Since $N_{8}+N_{8}^{\prime}+N_{8}^{\prime \prime}+N_{8}^{\prime \prime \prime} \simeq 12 E_{1}-6 e_{1} \simeq 6 L$ the curve $\pi\left(N_{8}\right)$ is a sextic $C_{8}$ in $\mathbb{P}^{2}$, which splits in all the double covers $Q, d P$ and $X_{2}$ of $\mathbb{P}^{2}$. The sextic $C_{8}$ is a rational curve (since it is the image of rational curves) and thus has 10 nodes. Moreover, since $C_{8}$ splits in the double covers, $C_{8} \cap C_{0}$ consists of 6 points with multiplicity two and $C_{8} \cap B$ consists of 12 points with multiplicity two. The inverse image of $C_{8}$ in $Q$ consists of two rational curves, one of bidegree $(1,5)$ which is the common image of $N_{8}$ and $N_{8}^{\prime \prime \prime}$ and one of bidegree $(5,1)$ which is the common image of $N_{8}^{\prime}$ and $N_{8}^{\prime \prime}$. The bidegrees of these curves are obtained by the fact that $N_{8}$ is a section of $E_{1}$ and a 5 -section of $E_{2}$, and $N_{8}^{\prime}$ is a section of $E_{1}$ and a 5 -section of $E_{2}$. The inverse image $q_{3}^{-1}\left(C_{8}\right)$ in $X_{1}$ consists of two rational curves, one is $\pi_{2}\left(N_{8}\right)=\pi_{2}\left(N_{8}^{\prime}\right)$ and it is the curve denoted by $\overline{N_{8}}$ in Section 4.3.2, the other is $\pi_{2}\left(N_{8}^{\prime \prime}\right)=\pi_{2}\left(N_{8}^{\prime \prime \prime}\right)$. 
Remark 4.14. As in the proof of Proposition 4.13 one is able to determine the image of the curves in the linear systems $\left|E_{i}\right|$ under the map $\pi$. The orbit of $E_{1}$ for $\left\langle\iota_{Q}^{*}, \iota_{d P}^{*}\right\rangle$ is $\left\{E_{1}, E_{2}\right\}$, thus we have two elliptic fibrations which are switched by $\sigma$ and by $\iota_{d P}$ but each of them is preserved by $\iota_{Q}$. Since $E_{1}+E_{2}=L$, a curve $F_{1} \in\left|E_{1}\right|$ is mapped to a line $f_{1}$ in $\mathbb{P}^{2}$. Moreover, for a general $F_{1}, q_{1}^{-1}\left(f_{1}\right)$ is the union of the two curves $\pi_{Q}\left(F_{1}\right)$ and $\pi_{Q}\left(\sigma\left(F_{1}\right)\right)$. Hence the line $f_{1}$ is tangent to the conic $C_{0}$ (which is the branch locus of $q_{1}: Q \rightarrow \mathbb{P}^{2}$ ). The line $f_{1}$ does not splits for the covers $q_{2}$ and $q_{3}$ and in particular the class $\left(q_{3}\right)_{*}\left(E_{1}\right)$ induces an elliptic fibration on $X_{1}$. So $q_{3}^{-1}\left(f_{1}\right)$ is a genus 1 curve. This implies that $q \cap B$ consists of 4 disjoint points (which are the branch points of the $2: 1$ cover $\left.q_{3}^{-1}\left(f_{1}\right) \rightarrow f_{1}\right)$. Hence the 1-dimensional linear system of genus 1 curve in $\left|E_{1}\right|$ is mapped by $\pi$ to the 1-dimensional linear system of lines tangent to the conic $C_{0}$. The same holds true for the 1-dimensional linear system $\left|E_{2}\right|$, since $\sigma^{*}\left(E_{1}\right)=E_{2}$.

By definition the 2: 1 map $q_{2}: d P \rightarrow \mathbb{P}^{2}$ is the anticanonical map, and then, denoted by $h$ the class of a line in $\mathbb{P}^{2}, q_{2}^{*}(h)=-K_{d P}$. So $q_{2}^{-1}\left(f_{1}\right)$ is a genus 1 curve in the anticanonical system, with the special property that it intersects the curve $q_{2}^{-1}\left(C_{0}\right)$ with even multiplicity in each of their intersection points. Hence the curve $q_{2}^{-1}\left(f_{1}\right) \subset d P$ splits in the double cover $\pi_{d P}: X_{2} \rightarrow d P$. With the notation of (4.5), the curve $\beta_{d P}\left(q_{2}^{-1}\left(f_{1}\right)\right)$ is a plane cubic tangent to $\beta_{d P}\left(q_{2}^{-1}\left(C_{0}\right)\right)$.

Until now our point of view was to consider $X_{2}$ as a surface with a symplectic automorphism $\sigma$ and to determine its structure as Nikulin surface, but one can consider the reverse problem: given a Nikulin surface with Néron-Severi group $M_{2,2}$, it has a very natural model as quartic in $\mathbb{P}^{3}$ with 8 nodes. To reconstruct the structure of this surface as double cover of $\mathbb{P}^{1} \times \mathbb{P}^{1}$ admitting a symplectic involution one has to identify the two elliptic fibrations $\varphi_{\left|E_{1}\right|}: X_{2} \rightarrow \mathbb{P}^{1}$ and $\varphi_{\left|E_{2}\right|}: X_{2} \rightarrow \mathbb{P}^{1}$. We gave a change of basis from $\left\{E_{1}, e_{1}, \ldots, e_{8}\right\}$ to $\left\{H, N_{1}, \ldots, N_{7}, \sum_{i=1}^{8} N_{1} / 2\right\}$ in proof of Proposition 4.12. Its inverse allows us to find the class of $E_{1}$ in terms of the classes $H$ and $N_{i}, i=1, \ldots, 8$, in particular $E_{1}=H-\left(\sum_{i=1}^{8} N_{i}\right) / 2$. The curves in this linear system are mapped to cubics by the linear system $\left|H-N_{8}\right|$. So, given a curve $F_{1} \in\left|E_{1}\right|, c:=\varphi_{\left|H-N_{8}\right|}\left(F_{1}\right)$ is a cubic curve in $\mathbb{P}^{2}$ and $\varphi_{\left|H-N_{8}\right|}^{-1}(c)$ consists of two curves, whose linear systems are $E_{1}=H-\left(\sum_{i=1}^{8} N_{i}\right) / 2$ and $E_{2}=$ $2 H-\left(\sum_{i=1}^{8} N_{i}\right) / 2-2 N_{8}$ respectively. Their sum exhibits $S$ as double cover of $\mathbb{P}^{1} \times \mathbb{P}^{1}$ admitting the required symplectic involution.

In [vGS, Section 3.7] an equation of $X_{2}$ is given, starting from a description of a K3 surface $X_{4}$ such that $\operatorname{NS}\left(X_{4}\right)=L_{4,2}^{\prime}$. The surface $X_{4}$ is given as complete intersections of three quadrics in $\mathbb{P}_{\left(y_{0}: y_{1}: x_{0}: \ldots: x_{3}\right)}^{5}$ of the form

$$
\left\{\begin{array}{l}
y_{0}^{2}=Q_{1}\left(x_{0}: x_{1}: x_{2}: x_{3}\right) \\
y_{1}^{2}=Q_{2}\left(x_{0}: x_{1}: x_{2}: x_{3}\right) \\
y_{0} y_{1}=Q_{3}\left(x_{0}: x_{1}: x_{2}: x_{3}\right)
\end{array}\right.
$$

Each complete intersection with equation (4.6) admits a symplectic involution induced by the projective transformation

$$
\left(y_{0}: y_{1}: x_{0}: x_{1}: x_{2}: x_{3}\right) \mapsto\left(-y_{0}:-y_{1}: x_{0}: x_{1}: x_{2}: x_{3}\right) .
$$

As shown in [vGS, Section 3.7], a singular model of the quotient surface is given by (4.7)

$$
Q_{1}\left(x_{0}: x_{1}: x_{2}: x_{3}\right) Q_{2}\left(x_{0}: x_{1}: x_{2}: x_{3}\right)=Q_{3}^{2}\left(x_{0}: x_{1}: x_{2}: x_{3}\right) \subset \mathbb{P}_{\left(x_{0}: x_{1}: x_{2}: x_{3}\right)}^{3} .
$$


By Proposition 4.5 the smooth model of the quotient surface (4.7) has Néron-Severi group isometric to $M_{2,2}$, i.e. it is the surface $X_{2} \simeq S$ and the map to $\mathbb{P}_{\left(x_{0}: x_{1}: x_{2}: x_{3}\right)}^{3}$ is given by the linear system of the pseudo ample polarization $H$ (with the notation of Proposition 4.12).

Let us consider the pencil of quadrics $\mathcal{P}_{t}:=\left\{Q_{1}=t Q_{3}\right\} \subset \mathbb{P}^{3}$. It cuts on $X_{2}$ a pencil of curves, whose class is $2 H-\sum_{i=1}^{8} N_{i}$, since all the quadrics in $\mathcal{P}_{t}$ pass through the 8 points in $Q_{1} \cap Q_{2} \cap Q_{3}$, which are the singular points of the surfaces in (4.7). For almost every $t, \mathcal{P}_{t}$ cuts two genus 1-curves on the surfaces in (4.7): one is the complete intersection $Q_{1} \cap Q_{2}$ (and does not depend on $t$ ) the other is $\left(Q_{1}-t Q_{3}\right) \cap\left(Q_{3}-t Q_{2}\right)$. So, the first curve is a fixed component of the linear system $2 H-\sum_{i=1}^{8} N_{i}$, the latter is a movable curve. The curves $Q_{1} \cap Q_{2}$ and $\left(Q_{1}-t Q_{3}\right) \cap\left(Q_{3}-t Q_{2}\right)$ intersect transversally in the singular points of the quartic (4.7). So, they have no intersection points in the blow up $X_{2}$ of the quartic (4.7) in its singular points. Hence the curves $Q_{1} \cap Q_{2}$ and $\left(Q_{1}-t Q_{3}\right) \cap\left(Q_{3}-t Q_{2}\right)$ are two fibers of the same fibration $X_{2} \rightarrow \mathbb{P}_{t}^{1}$ and are represented by the same divisor in $\operatorname{NS}\left(X_{2}\right)$. It is necessarily $\left(2 H-\sum_{i=1}^{8} N_{i}\right) / 2=H-\left(\sum_{i=1}^{8} N_{i}\right) / 2$. This is the divisor $E_{1}$ considered above so we conclude that if the surface $S \simeq X_{2}$ is embedded in $\mathbb{P}^{3}$ as a quartic of the form $Q_{1} Q_{2}=Q_{3}^{2}$, then the elliptic fibration $E_{1}$ is cut out by $\mathcal{P}_{t}:=\left\{Q_{1}=t Q_{3}\right\}$. The elliptic fibration $E_{2}$ is the image of $E_{1}$ under $\iota_{d P}$, which is the involution induced by the projection from the node $\varphi_{|H|}\left(N_{8}\right)$ of $\varphi_{|H|}\left(X_{2}\right)$.

4.5. Special 10-dimensional subfamilies of $\mathcal{L}_{2}$ and $\mathcal{M}_{2}$. In Proposition 3.1 we discussed the family $\mathcal{U}_{n}$ of $\left(U \oplus \mathbb{M}_{n}\right)$-polarized K3 surfaces, proving that it is contained in $\mathcal{L}_{n} \cap \mathcal{M}_{n}$ and it has codimension 1 in this space. This holds for every admissible $n$, so in particular for $n=2$. Here we reconsider this family, since it also has interesting properties with respect to the components of $\mathcal{M}_{2}$ : it is contained in the common intersection of all the irreducible components of $\mathcal{M}_{2}$. We discuss the analogous property for the components of $\mathcal{L}_{2}$, identifying another interesting family of K3 surfaces, which has codimension 1 in each component of $\mathcal{L}_{2}$. More precisely the aim of this section is to prove the following:

- There exists an irreducible connected 10-dimensional subvariety of the moduli space of $\mathrm{K} 3$ surfaces (it is $\mathcal{U}_{2}$ ) which is properly contained in all the families of Nikulin surfaces. Moreover all the K3 surfaces in this subvariety also admit a symplectic involution.

- There exists an irreducible connected 10-dimensional subvariety of the moduli space of K3 surfaces which is properly contained in all the families of K3 surfaces admitting a symplectic involution. All the K3 surfaces in this subvariety are also Nikulin surfaces.

Proposition 4.15. There exists an overlattice of index 2 of $U(2) \oplus N$, denoted by $(U(2) \oplus N)^{\prime}$, which is isometric to $U \oplus N$ and such that for any $d \in \mathbb{N}_{\geq 1}$, both the lattice $M_{d, 2}$ and the lattice $M_{2 d, 2}^{\prime}$ are primitively embedded in $(U(2) \oplus N)^{\prime}$. Hence all the irreducible components of the 11-dimensional families of Nikulin surfaces properly contain the 10-dimensional family $\mathcal{U}_{2}=\mathcal{P}(U \oplus N)$.

Proof. Let us consider the lattice $U(2) \oplus N$. Let $u_{1}$ and $u_{2}$ be the basis of $U(2)$ such that $u_{j}^{2}=0, j=1,2$ and $u_{1} u_{2}=2$, and let $w_{i, j}, i=1,2, j=1,2,3$, be a set of vectors in $N$ such that $w_{i, h} / 2$ are contained in the discriminant group of $N \subset U(2) \oplus N$. Moreover we assume that the discriminant form on $w_{i, j} / 2$, 
$i=1,2, j=1,2,3$ is $u(2)^{3}$. The vector $v:=\left(u_{1}+u_{2}+w_{1,1}+w_{2,1}\right) / 2$ is isotropic in $A_{U(2) \oplus N}$, and the lattice obtained by adding the vector $v$ to $U(2) \oplus N$ is an even overlattice of index 2 of $U(2) \oplus N$. Let us call it $(U(2) \oplus N)^{\prime}$. The discriminant group of $(U(2) \oplus N)^{\prime}$ is generated by $\left(u_{1}+w_{1,1}\right) / 2,\left(u_{1}+w_{1,2}\right) / 2, w_{i, j}, i=1,2$, $j=2,3$ and its discriminant form is $u(2)^{3}$. There is a unique, up to isometry, even hyperbolic lattice with rank 10, length 6 and prescribed discriminant form $u(2)^{3}$. Hence $(U(2) \oplus N)^{\prime} \simeq U \oplus N$.

To give a primitive embedding of $M_{d, 2} \simeq\langle 2 d\rangle \oplus N$ in $U \oplus N$ it suffices to give a primitive embedding of $\langle 2 d\rangle$ in $U$, for example the embedding $\left(\begin{array}{l}1 \\ d\end{array}\right) \hookrightarrow U$ is primitive.

To give a primitive embedding of $M_{2 d, 2}^{\prime}$ in $(U(2) \oplus N)^{\prime}$ we consider a primitive embedding of $M_{2 d, 2}$ in $U(2) \oplus N$, which extends primitively to their overlattices. As above, a primitive embedding of $M_{2 d, 2} \simeq\langle 4 d\rangle \oplus N$ in $U(2) \oplus N$ is induced by a primitive embedding of $\langle 4 d\rangle$ in $U(2)$. We fix this embedding to be $\left\langle u_{1}+d u_{2}\right\rangle \hookrightarrow$ $U(2)$. This induces a primitive embedding of $M_{2 d, 2}^{\prime}$ in $(U(2) \oplus N)^{\prime} \simeq U \oplus N$.

Let $S$ be a K3 surface with $\mathrm{NS}(S) \simeq U \oplus N$. Then $S$ admits an elliptic fibration with 8 reducible fibers of type $I_{2}$ and a 2 -torsion section. We denote by $F$ the class of the fiber of this fibration, by $O$ the class of the zero section, by $t$ the 2 -torsion section and by $C_{i}^{j}, i=0,1, j=1, \ldots, 8$ the $i$-th component of the $j$-th fiber (with the usual assumption that the 0-component meets the zero section). A basis of $U \oplus N$ is then given by $F, F+O, C_{1}^{j}, j=1, \ldots 7,\left(\sum_{j=1}^{8} C_{1}^{j}\right) / 2=2 F+O-t$. The translation by a 2-torsion section is a symplectic involution, denoted by $\sigma$ and classically called van Geemen-Sarti involution. Its action is $F \leftrightarrow F, O \leftrightarrow t$, $C_{1}^{j} \leftrightarrow C_{0}^{j}$. The sublattice of $\mathrm{NS}(S)$ invariant for $\sigma$ is $\operatorname{NS}(S)^{\sigma} \simeq\langle F, s+t\rangle \simeq U(2)$. This exhibits the Néron-Severi group $\operatorname{NS}(S) \simeq U \oplus N$ as an overlattice (necessarily of index $\left.2^{2}\right)$ of $U(2) \oplus E_{8}(-2)$, since $\left(\mathrm{NS}(S)^{\sigma}\right)^{\perp} \simeq E_{8}(-2)$. Chosen a positive integer $e$, the divisor $v:=F-e(s+t)$ has the following properties: $v^{2}=-4 e, v$ is invariant and $v^{\perp_{\mathrm{NS}(S)}} \simeq L_{2 e, 2}^{\prime}$. In particular the van Geemen-Sarti involution on $S$ induces the symplectic involution whose action on $\mathrm{NS}(S)$ is -1 on $E_{8}(-2) \hookrightarrow L_{2 e, 2}^{\prime} \simeq v^{\perp}$ and +1 on $v$. Hence the isometry $\sigma^{*}$ on $\operatorname{NS}(S)$ extends the isometry associated to the symplectic involution on $L_{2 e, 2}^{\prime}$-polarized K3 surfaces, once an embedding $L_{2 e, 2}^{\prime} \hookrightarrow(U \oplus N)$ as in the proof of Proposition4.15 is fixed.

Now we consider the analogous problem on the irreducible components of $\mathcal{L}_{n}$.

Proposition 4.16. The 10-dimensional family $\mathcal{P}\left(U \oplus E_{8}(-2)\right)$ is properly contained in all the families $\mathcal{P}\left(L_{e, 2}\right)$ and $\mathcal{P}\left(L_{2 e, 2}^{\prime}\right)$.

The lattice $U \oplus E_{8}(-2)$ is isometric to the lattice $U(2) \oplus N$, hence all the members of the family $\mathcal{P}\left(U \oplus E_{8}(-2)\right)$ are Nikulin surfaces.

Proof. The primitive embedding of $L_{e, 2} \simeq\langle 2 e\rangle \oplus E_{8}(-2)$ in $U \oplus E_{8}(-2)$ is induced by the primitive embedding of $\langle 2 e\rangle \simeq\left\langle\left(\begin{array}{l}1 \\ e\end{array}\right)\right\rangle$ in $U$, as in the proof of Proposition 4.15. We observe that $U \oplus E_{8}(-2)$ is an overlattice of index 2 of $U(2) \oplus E_{8}(-2)$. Indeed, similarly to what we did in proof of Proposition 4.15, we consider the basis $u_{1}$ and $u_{2}$ of $U(2) \subset U(2) \oplus E_{8}(-2)$ and the vectors $w_{i, j} / 2 i=1,2, j=1,2,3,4$ in $A_{U(2) \oplus E_{8}(-2)}$ such that the discriminant form on $u_{1} / 2, u_{2} / 2$ and $w_{i, j} / 2, i=1,2$, $j=1, \ldots, 4$ is $u(2)^{5}$. By adding $v=\left(u_{1}+u_{2}+w_{1,1}+w_{2,1}\right) / 2$ to $U(2) \oplus E_{8}(-2)$ 
one obtains an even overlattice $\left(U(2) \oplus E_{8}(-2)\right)^{\prime}$ of index 2 of $U(2) \oplus E_{8}(-2)$, which is isometric to $U \oplus E_{8}(-2)$. Hence the primitive embedding of $L_{2 e, 2}^{\prime}$ in $\left(U(2) \oplus E_{8}(-2)\right)^{\prime} \simeq U \oplus E_{8}(-2)$ is induced by a primitive embedding of $\langle 4 e\rangle$ in $U(2)$, which is given by $\langle 4 e\rangle \simeq\left\langle\left(\begin{array}{l}1 \\ e\end{array}\right)\right\rangle$ in $U(2)$.

Since $L_{e, 2}$ and $L_{2 e, 2}^{\prime}$ are primitively embedded in $U \oplus E_{8}(-2)$ and they determine uniquely their orthogonal complement in $\Lambda_{K 3}$, the families $\mathcal{P}\left(L_{e, 2}\right)$ and $\mathcal{P}\left(L_{2 e, 2}^{\prime}\right)$ properly contain the family $\mathcal{P}\left(U \oplus E_{8}(-2)\right)$.

The isometry between the lattices $U \oplus E_{8}(-2)$ and $U(2) \oplus N$ follows by observing that they are lattices with rank 10, length 8 and the same discriminant form.

Let $\mathcal{E}_{R}: R \rightarrow \mathbb{P}^{1}$ be a rational elliptic surface (i.e. $R$ is a rational surface endowed with an elliptic fibration $\mathcal{E}_{R}$ ). It is known that a base change of order 2 on this elliptic fibration branched on two smooth fibers induces an elliptic fibration $\mathcal{E}_{S}: S \rightarrow \mathbb{P}^{1}$ on a K3 surface $S$. If the fibration $\mathcal{E}_{R}$ has no reducible fibers, then $\mathrm{NS}(S) \simeq U \oplus E_{8}(-2)$, see e.g. GSal, Proposition 4.6]. More in general the family of the K3 surfaces obtained by a base change of order 2 on a rational elliptic surface, is the family $\mathcal{P}\left(U \oplus E_{8}(-2)\right)$, see e.g. GSal].

Proposition 4.17. The 10-dimensional family $\mathcal{P}\left(U \oplus E_{8}(-2)\right)$ is the family $\mathcal{R}$ of the $K 3$ surfaces obtained by a base change of order two on a rational elliptic fibration $\mathcal{E}_{R}: R \rightarrow \mathbb{P}^{1}$. Let $S$ be a general member of $\mathcal{R}$ and let $\mathcal{E}_{S}$ be the elliptic fibration induced by $\mathcal{E}_{R}: \mathcal{E}_{S}$ has no reducible fibers and its Mordell-Weil rank is equal to 8. The symplectic involution $\sigma$ on $S$ preserves $\mathcal{E}_{S}$. Denoted by $\widetilde{S / \sigma}$ the desingularization of $S / \sigma, \mathrm{NS}(\widetilde{S / \sigma}) \simeq U \oplus D_{4} \oplus D_{4}$.

Proof. We already observed that $S$ is obtained by a base change of order 2 by $\mathcal{E}_{R}: R \rightarrow \mathbb{P}^{1}$. Then $\mathcal{E}_{S}$ admits an involution $\iota$ which acts only on the basis of the fibration, and which is the deck involution of the generically $2: 1$ cover $R \rightarrow S$. The involution $\iota$ maps fibers of $\mathcal{E}_{S}$ to other fibers and in particular preserves the class of the fiber and of the sections, i.e. it acts trivially on the Néron-Severi group. Thus it preserves the elliptic fibration (cf. GSal, Proposition 4.6]). Moreover, $\iota$ preserves exactly two fibers of $\mathcal{E}_{S}$ (the ramification fibers of the cover $R \rightarrow S$ ). The elliptic fibration $\mathcal{E}_{S}$ is preserved also by the elliptic involution $\epsilon$, which preserves the classes of the fiber and of the zero section (i.e. a set of generators of $U$ in $\left.\mathrm{NS}(S) \simeq U \oplus E_{8}(-2)\right)$. The composition $\sigma:=\iota \circ \epsilon$ is a symplectic involution which acts trivially on $U \hookrightarrow U \oplus E_{8}(-2) \simeq \mathrm{NS}(S)$ and as - id on $E_{8}(-2) \hookrightarrow U \oplus E_{8}(-2) \simeq$ $\mathrm{NS}(S)$. Thus $\sigma$ is a symplectic involution whose fixed locus consists of 4 points on each of the two fibers preserved by $\iota$. Hence the elliptic fibration $\mathcal{E}_{S}: S \rightarrow \mathbb{P}^{1}$ induces an elliptic fibration on $\widetilde{S / \sigma}$ whose generic fiber is a copy of the two fibers of $\mathcal{E}_{S}$ switched by $\sigma$. The images of the two fibers preserved by $\iota$ are two fibers of type $I_{0}^{*}$. The Picard number of $\widetilde{S / \sigma}$ is 10 , which is also the rank of the trivial lattice of an elliptic fibration with two fibers of type $I_{0}^{*}$. We conclude that there are no sections of infinite order for the elliptic fibration induced by $\mathcal{E}_{S}$ on $\widetilde{S / \sigma}$ and that $\mathrm{NS}(\widetilde{S / \sigma}) \simeq U \oplus D_{4} \oplus D_{4}$.

We observe that $U \oplus D_{4} \oplus D_{4} \not \varkappa U \oplus E_{8}(-2)$ since their discriminant groups are different, so $\mathrm{NS}(S) \not \mathrm{NS}(\widetilde{S / \sigma})$. 
By Proposition 4.16, if $S$ is a K3 surface such that $\mathrm{NS}(S) \simeq U \oplus E_{8}(-2)$, then it admits a symplectic involution (described in the proof of Proposition 4.17) and it is also $2: 1$ cyclically covered by a K3 surface. So it admits a 2-divisible set of rational curves, which we describe here. As observed $S$ is obtained by a base change of order 2 on $R$. Since $R$ is a rational elliptic surface, it is the blow up of $\mathbb{P}^{2}$ in nine points which are the base points of a pencil of generically smooth cubics. So $S$, which is a $2: 1$ double cover of $R$ branched on two smooth fibers, is a generically $2: 1$ cover of $\mathbb{P}^{2}$ branched in the union of two smooth cubics $C_{1}$ and $C_{2}$ (see e.g. GSal, Section 2.2]). The branch locus is singular in the nine points $C_{1} \cap C_{2}$. We denote by $H$ the genus 2 divisor on $S$ such that $\varphi_{|H|}: S \rightarrow \mathbb{P}^{2}$ is this $2: 1$ cover of $\mathbb{P}^{2}$ and by $D_{i}, i=0, \ldots 8$, the classes of the rational curves contracted by $\varphi_{|H|}$ to the nine singular points of the branch locus. By construction the fiber of the fibration $\mathcal{E}_{S}$ is the class of $C_{1}$ (and of $C_{2}$ ), i.e. $\left(3 H-\sum_{i=0}^{8} D_{i}\right) / 2$. The curves in the linear system $\left|H-D_{0}\right|$ (and in $\left|H-D_{1}\right|$ respectively) on $S$ are mapped to lines of a pencil in $\mathbb{P}^{2}$, with base point $\varphi_{|H|}\left(D_{0}\right)$ (with base point $\varphi_{|H|}\left(D_{1}\right)$ respectively). Each line in this pencil meets the branch in 4 points (with the exception of $\varphi_{|H|}\left(D_{0}\right)$ ), and so its inverse image in $S$ is a $2: 1$ cover of a rational curve branched in 4 points. So the curves in $\left|H-D_{0}\right|$ (resp. $\left|H-D_{1}\right|$ ) are genus 1 curves and $\left|H-D_{0}\right|$ and $\left|H-D_{1}\right|$ induce two genus 1 fibrations on $S$ (see GSal, Proposition 3.8]). Since $\left(H-D_{0}\right)\left(H-D_{1}\right)=2$, the map $\varphi_{\left|2 H-D_{0}-D_{1}\right|}$ is a generically $2: 1$ map to $\mathbb{P}^{1} \times \mathbb{P}^{1} \subset \mathbb{P}^{3}$ (see [SD]). It contracts the 8 mutually disjoint rational curves $D_{i}, i=2, \ldots, 8$, and $H-D_{0}-D_{1}$. The last contracted curve is the pullback of the line through the two points $\varphi_{|H|}\left(D_{0}\right)$ and $\varphi_{|H|}\left(D_{1}\right)$. The $2: 1$ map $\varphi_{\left|H-D_{0}\right|} \times \varphi_{\left|H-D_{1}\right|}: S \rightarrow \mathbb{P}^{1} \times \mathbb{P}^{1}$ is induced by the $2: 1$ map $\varphi_{|H|}: S \rightarrow \mathbb{P}^{2}$ via the birational transformation $\beta: \mathbb{P}^{2} \rightarrow \mathbb{P}^{1} \times \mathbb{P}^{1}$, which is the blow up of $\mathbb{P}^{2}$ in the two points $\varphi_{|H|}\left(D_{0}\right)$ and $\varphi_{|H|}\left(D_{1}\right)$ followed by the contraction of the line through these points. So the branch locus of the $2: 1$ cover $S \rightarrow \mathbb{P}^{1} \times \mathbb{P}^{1}$ splits in the union of two genus 1 curves of bidegree $(2,2)$, which are the images of the two cubics $C_{1}$ and $C_{2}$ under the birational transformation $\beta$. The curves $\beta\left(C_{1}\right)$ and $\beta\left(C_{2}\right)$ intersect in 8 points in $\mathbb{P}^{1} \times \mathbb{P}^{1}$, which are the images of the curves $D_{i}, i=2, \ldots, 8$, and $H-D_{0}-D_{1}$. The classes of the pullback of $\beta\left(C_{1}\right)$ and $\beta\left(C_{2}\right)$ on $S$ coincide and each of them is represented by the class $\left(2\left(H-D_{0}\right)+2\left(H-D_{1}\right)-\sum_{i=2}^{8} D_{i}-\left(H-D_{0}-D_{1}\right)\right) / 2$. So the set of curves $\left\{D_{2}, \ldots D_{8}, H-D_{0}-D_{1}\right\}$ is an even set.

\section{REFERENCES}

[BSV] S. Boissière, A. Sarti, D.C. Veniani, On prime degree isogenies between K3 surfaces, Rend. Circ. Mat. Palermo (2) 66 (2017), 3-18.

$[\mathrm{Bu}] \quad$ N. Buskin, Every rational Hodge isometry between two K3 surfaces is algebraic, Journal für die reine und angewandte Mathematik (2017), doi:10.1515/crelle-2017-0027.

[C] F. Catanese, On the moduli spaces of surfaces of general type, J. Differential Geom. 19 (1984), 483-515.

[DO] I. Dolgachev, D, Ortland Point sets in projective spaces and theta functions, Astérisque 165 (1988).

[G1] A. Garbagnati, K3 surfaces with abelian and dihedral groups of symplectic automorphisms, Comm. Algebra 41 (2013), 583-616.

[G2] A. Garbagnati, On K3 surface quotients of K3 or Abelian surfaces, Canad. J. Math. 69 (2017), 338-372.

[GSal] A. Garbagnati, C. Salgado, Linear systems on rational elliptic surfaces and elliptic fibrations on K3 surfaces, J. Pure Appl. Algebra 223 (2019), 277-300. 
[GSar1] A. Garbagnati, A. Sarti, Projective models of K3 surfaces with an even set, Adv. Geometry 8 (2008), 413-440.

[GSar2] A. Garbagnati,A. Sarti Symplectic automorphisms of prime order on K3 surfaces, J. Algebra 318 (2007), 323-350.

[GSar3] A. Garbagnati, A. Sarti, Elliptic fibrations and symplectic automorphisms on K3 surfaces, Comm. Algebra 37 (2009), 3601-3631.

[vGS] B. van Geemen, A. Sarti, Nikulin involutions on K3 surfaces, Math. Z. 255 (2007), 731-753.

[H] K. Hashimoto, Finite symplectic actions on the K3 lattice, Nagoya Math. J., Volume 206 (2012), 99-153.

[Huy] D. Huybrechts, Motives of isogenous K3 surfaces, to appear in Commentarii Mathematici Helvetici (2017).

[I] H. Inose, Defining equations of singular K3 surfaces and a notion of isogeny. Proc. int. Symp. on algebraic geometry, Kyoto, 495-502, 1977.

[L] R. Laterveer, A family of K3 surfaces having finite-dimensional motive, Arch. Math. (Basel) 106 (2016), no. 6, 515-524.

$[\mathrm{MM}] \quad$ R. Miranda, D.R. Morrison, Embeddings of Integral Quadratic Forms, preprint available at https://www.math.colostate.edu// miranda/preprints/eiqf.pdf

[Mo] D.R. Morrison, On K3 surfaces with large Picard number, Invent. Math. 75 (1984) 105121 .

[M] S. Mukai, On the moduli space of bundles on K3 surfaces, I. In: Vector Bundles on Algebraic Varieties, Bombay (1984), 341-413.

[N1] V.V. Nikulin, Integer symmetric bilinear forms and some of their geometric applications, Izv. Akad. Nauk SSSR Ser. Mat. 43 (1979), no. 1, 111-177.

[N2] V.V. Nikulin, Finite automorphism groups of Kähler K3 surfaces. Trans. Moscow Math. Soc. 38 (1980), 71-135.

[N3] V. Nikulin, On correspondences between surfaces of K3 type, Math. USSR-Izv. 30 (1988), 375-383.

[P] C. Pedrini, Bloch's conjecture and valences of correspondences for K3 surfaces, arXiv: $1510.05832 \mathrm{v} 1$.

[SD] B. Saint-Donat, Projective Models of K3 surfaces, Amer. J. of Math. 96 (1974) 602-639.

[Ša] I. Šafarevič, Le théorème de Torelli pour les surfaces algébriques de type K3, Actes du Congres International des Mathématiciens (Nice, 1970), Gauthier-Villars, Paris, (1971), 413-417.

[Sh] I. Shimada, On elliptic K3 surfaces, Michigan Math. J. 47 (2000), 423-446, arXiv version with the complete Table arXiv:math/0505140

Chiara Camere, Dipartimento di Matematica, Università degli Studi di Genova, Via Dodecaneso 35, 16146 Genova (GE)

E-mail address: camere@dima.unige.it

Alice Garbagnati, Università degli Studi di Milano, Dipartimento di Matematica, via Cesare Saldini 5020133 Milano, Italy

E-mail address: alice.garbagnati@unimi.it

$U R L:$ https://sites.google.com/site/alicegarbagnati/ 